\title{
Exosome-Mediated miR-7-5p Delivery Enhances the Anticancer Effect of Everolimus via Blocking MNK/elF4E Axis in Non-Small Cell Lung Cancer
}

Sile Liu

Second Xiangya Hospital Department of Pathology

Weiyuan Wang

Xiangya Hospital Central South University

Yue Ning

Second Xiangya Hospital Department of Pathology

Hongmei Zheng

Second Xiangya Hospital Department of Pathology

\section{Yuting Zhan}

Second Xiangya Hospital Department of Pathology

\section{Haihua Wang}

Second Xiangya Hospital Department of Pathology

Yang Yang

Second Xiangya Hospital Department of Pathology

Jiadi Luo

Second Xiangya Hospital Department of Pathology

Qiuyuan Wen

Second Xiangya Hospital Department of Pathology

Hongjing Zang

Second Xiangya Hospital Department of Pathology

\section{Jinwu Peng}

Xiangya Hospital Central South University

Jian Ma

Central South University Xiangya School of Medicine

Songqing Fan ( $\nabla$ songqingfan@csu.edu.cn )

The Second Xiangya Hospital, Central South University https://orcid.org/0000-0002-1486-9909

\section{Research}

Keywords: mTOR, Everolimus, exosome, miR-7-5p, MNK/elF4E axis, apoptosis

Posted Date: September 17th, 2021

DOI: https://doi.org/10.21203/rs.3.rs-880087/v1

License: (c) (i) This work is licensed under a Creative Commons Attribution 4.0 International License. Read Full License 
Version of Record: A version of this preprint was published at Cell Death \& Disease on February 1st, 2022. See the published version at https://doi.org/10.1038/s41419-022-04565-7. 


\section{Abstract \\ Background}

Everolimus is a kind of mTOR inhibitors. Activated mitogen-activated protein kinase interacting kinases/eukaryotic translation initiation factor 4E (MNK/elF4E) axis plays a crucial role in resistance to Everolimus in non-small cell lung cancer (NSCLC). Typically, eIF4E phosphorylation increased by mTOR inhibitors was mainly mediated by MNKs. But the mechanisms are poorly understood. Recently, extensive reprogramming of miRNA profiles has also been found after long-term mTOR inhibitor exposing. Our previous studies have confirmed that tumor suppressor miR-7-5p was decreased in A549 cells after treatment with Everolimus. Exactly, MNK1 is the target of miR-7-5p. Here, we investigated the biological functions and potential molecular mechanisms of miR-7-5p in the NSCLC undergoing treatment with Everolimus.

\section{Methods}

miR-7-5p level and expression of main markers of MNK/elF4E axis were evaluated by qRT-PCR, western blot, in situ hybridization, and immunohistochemistry on NSCLC cell lines and human NSCLC samples. Proliferation, migration and invasion of NSCLC cells in culture were explored by Colony formation, CCK-8, Wound healing and Transwell assays. NSCLC cell tumorigenicity was assessed by xenotransplants in nude mice. Targeted binding of miR-7-5p to MNK1 was confirmed by the Dual-luciferase reporter assay. And the isolation and identification of exosomes were investigated by Invitrogen ${ }^{\text {TM }}$ Total Exosome RNA Isolation Kit, western blot, transmission electron microscopy and Zetasizer Nano ZS90 instrument.

\section{Results}

Everolimus targeted mTORC1 inducing NSCLC cells to secrete miR-7-5p-loaded exosomes in Rab27A and Rab27B dependent manners. Loss of intracellular miR-7-5p induced phosphorylation of MNK/elF4E axis, but supplement of extra exosomal miR-7-5p could reverse it. Of note, both lower expression of miR-7-5p and elevated MNK1 protein were associated with poor prognosis of NSCLC. Both endogenous miR-7-5p and exo-miR-7-5p enhanced the therapeutic efficacy of Everolimus through inhibiting the proliferation, migration, and metastasis of NSCLC in vitro and vivo. Combination of miR-7-5p with Everolimus induced apoptosis to exhibit a synergistic anticancer therapeutic efficacy via dual abrogation of MNK/elF4E and mTOR in NSCLC.

\section{Conclusion}

Everolimus decreases the intracellular miR-7-5p levels through release of miR-7-5p loaded exosomes from NSCLC cells in Rab27A and Rab27B dependent manners. Either endogenous miR-7-5p or exo-miR-7-5p combined with Everolimus can enhance the anticancer efficacy via targeting MNK/elF4E axis and mTOR. Therefore, exosome-mediated miR-7-5p delivery combined with Everolimus may be considered as a promising novel combined therapeutic strategy for NSCLC.

\section{Background}

The mammalian target of rapamycin (mTOR) is a serine/threonine kinase that can regulate cell growth and proliferation under physiological and pathological conditions. Dysregulation of the mTOR signaling pathway can be seen in many cancers including non-small cell lung cancer (NSCLC). Furthermore, abnormal activation of PI3K-AKT-mTOR pathway 
have been proved to generate acquired resistance to epidermal growth factor receptor (EGFR) tyrosine kinase inhibitors (TKIs) in NSCLC[1]. The functions of mTOR mainly exercise by forming two different complexes, named mTOR complex 1 (mTORC1) and mTOR complex 2 (mTORC2)[2]. Everolimus (RAD001) is an effective mTORC1 inhibitor to be a cuttingedge drug for targeted therapy, but has poor efficacy as a monotherapy[3]. In vitro studies suggested the clinical efficacy of combining Everolimus with EGFR-TKIs in TKIs-resistant NSCLC cell lines[4]. But the subsequent clinical trials showed limited effect of combination therapy in unselected NSCLC patients[5]. Thus, it is worth to further explore the potential effect of Everolimus in NSCLC patients. Similar to other anti-tumor treatments, long-term Everolimus treatment can lead to secondary drug resistance caused by genomic instability[6]. Activation of other regulatory proteins or survival cascades are also involved in resistance to Everolimus, like mitogen-activated protein kinase (MAPK)-interacting kinases (MNK) 1 and 2. The activated MNKs are phosphorylated, which continuously phosphorylate elF4E, and play a crucial role in mediating resistance to rapamycin in NSCLC [7]. Moreover, elF4E phosphorylation increased by mTOR inhibitors was mainly mediated by MNKs [8]. However, the mechanisms by which Everolimus induced phosphorylation of MNK/elF4E axis are poorly understood.

Totary-Jain et al. demonstrated that long-term treatment with mTOR inhibitor rapamycin showed extensive reprogramming of miRNA profiles, characterized by down-regulation of tumor suppressor miRNAs. But delivery of tumor suppressor miRNAs would restore the sensitivity to rapamycin [9]. Our previous studies have found that when Everolimus treated on NSCLC cells, its tumor suppressor effect stabilized at a concentration of 25nM, accompanied by activation of the MNK/elF4E axis[10]. Besides, we carried miRNA array out to detect changes of the miRNA profiles in A549 cells accepting Everolimus treatment [11]. The down-regulated miR-7-5p has attracted our attention. For the reasons that numerous reports confirmed miR-7 is a miRNA involved in the regulation of several signaling pathways, and acts as a tumor suppressor in NSCLC[12]. It dominantly regulates several basic cellular processes, which include proliferation, differentiation, apoptosis, migration and expression of stem cell features [13]. It is worth noting that miR-7 was also shown to decrease EGFR mRNA expression[14] and regulate many genes of the mTOR pathway, like MNK, elF4E and $70 \mathrm{kDa}$ ribosomal protein S6 kinase (p70S6K) [15]. And miR-7 has yet to be discovered in terms of the possibility of using it as a specific biotherapeutic agent in NSCLC.

Exosomes derived from tumor cells carry the components of membranes and cells fall off into the extracellular space and cause the loss of intracellular proteins, miRNAs and IncRNAs via the secretion. It reflects the phenotype of the original cells, the effect of drug treatments on cells and the process of specific sorting of these molecules by tumor cells. Therefore, it is of great value for the early diagnosis of disease, the assessment of pathogen burden or disease stage, and the monitoring of the response to treatment[16]. This secretion-dependent mechanism is regulated by mTORC1[17]. So, when Everolimus targeted mTORC1, the occurrence of resistance mechanism might relate to the release of exosomes.

Above all prompted that Everolimus inhibiting mTORC1 might alter the level of miR-7-5p in NSCLC in an exosomedependent manner, thereby activating MNK /elF4E axis, which involved in the resistance to Everolimus, but have not been studied yet. Therefore, in our study, we focused on the new mechanism of miR-7-5p in Everolimus resistance and looked for new treatment options in NSCLC. Here, we found that when the activity of mTOR was inhibited by Everolimus in NSCLC, it would promote the secretion of exosomes derived from tumor cells, which loaded miR-7-5p. Consequently, MNK/elF4E axis was activated followed by intracellular miR-7-5p declining, thereby limiting the tumor suppressor effect of Everolimus. Hopefully, endogenous miR-7-5p or exo-miR-7-5p could synergistically enhance the anticancer efficacy of Everolimus by inducing apoptosis via dual abrogation of MNK/elF4E axis and mTOR.

\section{Materials And Methods}

We describe antibodies, reagents and cells in details in the Supplemental Material and Methods. 


\section{Clinical data and tissue microarrays (TMA)}

In this study, a total of 318 cases of NSCLC and 91 cases of non-cancerous lung tissue (Non-CLT) were chosen randomly from The Second Xiangya Hospital of Central South University (Changsha, China). Complete clinical records and followup data were available for all patients. Besides, 40 pairs of primary NSCLC tissues and adjacent non-tumor lung tissues were collected from 40 cases of NSCLC. Protocols were approved by The Second Xiangya Hospital of Central South University Ethics Review Board (Scientific and Research Ethics Committee, No. K021/2021). All patients involved in our study had written informed consent. The staging classification of this research was carried out based on the criteria of the 8th edition of the AJCC/UICC TNM staging system of lung cancer (2017). The confirmed histological diagnosis of NSCLC according to the World Health Organization histological classification of lung cancer. We used the TMA technology designed and constructed high-throughput NSCLC TMAs according to rules previously described [18].

\section{Quantitative PCR analysis}

We extracted total RNA using TRIzol reagent (Invitrogen). Two $\mu \mathrm{g}$ of total RNA was performed to synthesize cDNA using by Mir-X miRNA First-Strand Synthesis Kit (Takara) or RevertAid First-Strand cDNA Synthesis Kit (Thermo) according to the manufacturer's protocol. The RNA level was measured by qPCR with a TB Green premix Ex Taq II kit (Takara) or $2 \times$ SYBR Green qPCR Master Mix (Bimake) on CFX96 Real-Time PCR Detection System (Bio-Rad). Relative expression was determined with a U6 control or $\beta$-actin through the $2^{-\Delta \Delta}$ Ct method.

Before isolation of RNA from exosomes, $1 \times 10^{8}$ copies/ $\mu \mathrm{L}$ of C. elegans cel-miR-39-3p mimics (Qiagen) was added to samples as a spike-in control.

\section{Western blotting}

Whole-cell protein lysates preparation and Western blotting analysis were performed as described previously [19]. Cytoplasmic and nuclear extracts were obtained by using the CEB-NEB kit (Applygen, China) according to the manufacturer's protocol.

\section{Exosome isolation and identification}

Before cell culture, FBS was depleted of exosomes by ultracentrifugation at $1 \times 10^{5} \mathrm{~g}$ at $4{ }^{\circ} \mathrm{C}$ for $12 \mathrm{~h}$, then the supernatant was harvested and filtered with a 0.22 um filter (Millipore). After $24 \mathrm{~h}$, the cell culture medium with exosomefree FBS was collected and removed floating cells and cellular debris from the supernatant by centrifuging at $3000 \mathrm{~g}$ for $30 \mathrm{~min}$. The supernatant was then passed through a $0.22 \mu \mathrm{m}$ filter. The resultant supernatant was forced through the membrane by centrifugal filtration at $3000 \mathrm{~g}$ for $1 \mathrm{~h}$ with the ultrafiltration device (UFC900396, Millipore). Finally, the total exosome isolation reagent (from cell culture media, Invitrogen) was added to the concentrated solution at a ratio of 1:2. Subsequent exosome extraction was conducted according to the manufacturer's protocol. The exosomes were used for the following experiments immediately or stored at $-80^{\circ} \mathrm{C}$. Exosomal protein was measured by the $\mathrm{BCA}^{\mathrm{Tm}}$ Protein Assay Kit (Thermo). The concentration of exosomal proteins was quantified using a BCA protein assay kit (Keygen Biotech, KGP902, China). HSP70, TSG101, CD63 and CD9 expression were measured using western blot analysis, and GAPDH as an internal reference.

Isolated exosomes were fixed in $1 \%$ glutaraldehyde for $10 \mathrm{~min}$, washed with deionized water. $10 \mu \mathrm{L}$ of exosome suspension was placed on formvar carbon-coated 300-mesh copper electron microscopy grids, incubated for 5 min at room temperature and air-dried for $5 \mathrm{~min}$. Images were obtained by TEM[20]. In addition, exosomes were examined via injecting into the Zetasizer Nano ZS90 instrument. Phosphate buffered saline was used as controls. Data was analyzed by Zetasizer software.

\section{Exosome labeling}


Exosomes were resuspended in $500 \mu \mathrm{L}$ PBS mixed with $1 \mu \mathrm{L}$ of the $500 \times$ labeling dye (ExoGlow protein EV Labeling Kit, SBI) and incubated at $37^{\circ} \mathrm{C}$ for $30 \mathrm{~min}$. Then $167 \mu \mathrm{L}$ ExoQuick-TC (SBI) was added to stop the reaction and incubated at $4{ }^{\circ} \mathrm{C}$ overnight. Labeled exosomes were isolated from the supernatant by centrifugation of $10000 \mathrm{rpm}$ for $30 \mathrm{~min}$ and resuspended in PBS.

Fluorescently labeled exosomes were added to A549 cells which were at $80 \%$ confluence and incubated overnight. Cells were fixed with $4 \%$ paraformaldehyde for $30 \mathrm{~min}$ at room temperature and then permeabilized with $0.25 \%$ Triton-X for 30 min. Next, the cells were stained with TRITC phalloidin for 30 min and 4', 6-diamidino-2-phenylindole (DAPI) for 5 min sequentially. Finally, cells were washed twice with PBS to remove excess DAPI. A549 cells that up took the labeled exosomes were observed under a fluorescence microscope.

\section{Cell transfection}

The synthetic miRNA mimics, inhibitors, negative control (NC), siTSC1, siTSC2, siRab27A or siRab27B (GenePharma, Shanghai, China) were mixed with lipofectamine 3000 (Invitrogen) and then added to the cell culture medium according to the manufacturer's instructions. After $24 \mathrm{~h}$ or $48 \mathrm{~h}$ of transfection, total RNA and protein were extracted from cells, respectively.

\section{Lentivirus transduction}

The LV-miR-7-5p and the control virus LV-NC were purchased from GenePharma (Shanghai, China). Cells were infected with lentivirus for 2 days and then added to puromycin $(2.5 \mathrm{mg} / \mathrm{mL})$ for 2 weeks to generate stably transduced cell lines.

\section{Dual-luciferase reporter assay}

The full length of MNK1 3'-UTR were amplified and subcloned into the pmirGLO dual-luciferase miRNA target expression vector. The wild type and mutant sites in MNK1 3'-UTR or elF4E 3'-UTR were produced by GeneCreate Biotech (Wuhan, China). Luciferase activity assays were performed with the Dual-Luciferase Reporter Assay System (Promega, Madison, $\mathrm{WI})$.

\section{Xenograft Mouse Tumor Models}

For the subcutaneous tumor model, the LV-miR-7-5p and LV-NC A549 cells ( 1 x 10 ${ }^{7}$ cells) in $200 \mu \mathrm{L}$ serum-free medium were injected subcutaneously into female BALB/c nude mice, respectively. As soon as the tumor volume reached 50$100 \mathrm{~mm}^{3}$, mice would be divided into four groups ( $\mathrm{n}=6$ /group) named as the LV-NC group, the LV-miR-7-5p group, the LV-NC combined Everolimus ( $3 \mathrm{mg} / \mathrm{kg} /$ day, oral gavage) group and the LV-miR-7-5p combined Everolimus group. The four groups received indicated treatments. The size of the tumors and the weight of the mice were monitored every 2 days. After 28 days, the mice were euthanized and the tumors were obtained. Tumor volume was calculated with the formula: $V=($ length $\times$ width2) $/ 2$. Finally, all tumors were excised, weighed, and fixed in $10 \%$ neutral buffered formalin for $24 \mathrm{~h}$, and the paraffin sections were routinely stained with hematoxylin/eosin.

As for the intraperitoneal tumor model, single-cell suspensions of luciferase tagged A549-cells $\left(1 \times 10^{7}\right.$ cells $)$ were injected into the abdominal of female BALB/c nude mice. And the baseline level of tumor cells was monitored by live imaging technology immediately after injection. And then, the survival and mental state of the nude mice were monitored every two days. After one week, we confirmed the survival of tumor tissue, randomly divided them into 6 groups ( $n=3$ /group), and received PBS, exo-NC, exo-miR-7-5p, Everolimus, exo-NC combined with Everolimus and exomiR-7-5p combined with Everolimus treatment, respectively. The development of tumor burden was monitored by bioluminescence imaging (BLI) of anesthetized mice that were intraperitoneally injected with $75 \mathrm{mg} / \mathrm{kg}$ D-luciferin (Thermo). 


\section{Colony formation assay, CCK-8 assay, Wound healing and Transwell migration assays}

These procedures were performed as previously described[11].

\section{Flow cytometry}

For cell apoptosis detection, cells were plated into 6-well plates and transfected with miRNA mimics for $24 \mathrm{~h}$ and treated with/without $25 \mathrm{nM}$ Everolimus after an additional $48 \mathrm{~h}$. The number of apoptotic cells were detected using a FITCAnnexin $\mathrm{V}$ apoptosis detection kit (BD Pharmingen) and a flow cytometer following the manufacturer's instructions.

\section{Immunohistochemistry (IHC) \& In-situ hybridization (ISH)}

The immunohistochemical staining for Ki-67, p-elF4E $\mathrm{E}^{\mathrm{S} 209}$ and MNK1 and the In-situ hybridization assay of miR-7-5p was performed as previously described[11, 21]. And the expression levels of Ki-67, miR-7-5p, p-elF4E ${ }^{\mathrm{S} 209}$ and MNK1 in tissues of xenograft mouse tumor models were analyzed by the Image J Software.

\section{Statistical analysis}

Statistical analyses were determined by chi-square test, Spearman correlation test, multivariate Cox regression analysis, Pearson correlation, Wilcoxon rank-sum test, or student's t-test as appropriate using SPSS 19.0 and GraphPad Prism 5.0. Error bars indicated the standard deviation in all the figures. ${ }^{*} P<0.05, * \star P<0.01, * \star \star P<0.001$. And $P$ value $<0.05$ was considered significant.

\section{Results}

\section{Everolimus targeted mTORC1 inducing NSCLC cells to secrete miR-7-5p-loaded exosomes in Rab27A and Rab27B dependent manners}

In multiple NSCLC cell lines, including A549, H358, H520 and SPC-A1, the level of miR-7-5p was significantly downregulated after treatment with Everolimus (Fig. 1A). And it lasted more than 48 hours without changing of autophagy in NSCLC cell lines (Figure S1A). In order to find the reason for the reduction of miR-7-5p, we further analyzed the lung squamous cell carcinoma mRNA data of TCGA, and divided the data into two groups named mTOR ${ }^{\text {low }}$ group and $m T O R^{\text {high }}$ group according to the mean value of mTOR level in the samples. The GSEA on the mRNA profiles changes revealed negative associations between mTOR and gene sets involved in extracellular exosomes (Fig. 1B). Consequently, we focused on the exosomes derived from NSCLC cells in the culture medium. They were isolated and identified by transmission electron microscope (TEM) (Fig. 1C) and NanoSight Analysis (Fig. 1D). The size and morphology of exosomes were similar between two groups, presented as 50-100nm round like. It was also found the fluorescent labelled exosomal markers CD63 and HSP70 in the cytoplasm was significantly weakened after Everolimus treating (Fig. 1E). Meanwhile, expression of exosomal markers HSP70, TSG101, CD63 and CD9 significantly enriched in exosomes accompanied by down-regulation of intracellular exosomal markers in A549 cells by Western blotting (Fig. 1F). To further determine whether there were association between the declination of intracellular miR-7-5p and elevation of release of exosomes induced by Everolimus, we used exosomes inhibitor GW4869 to block the release of exosomes. When the secretion of exosomes was inhibited by GW4869, miR-7-5p distribution was reversed (Fig. 1G), which indicated that Everolimus induced the secretion of miR-7-5p-loaded exosomes into the surrounding extracellular environment, resulting in the loss of miR-7-5p in intracellular.

Furthermore, we performed siTSC1/2 to active [22] but Everolimus to inhibit the activity of mTORC1. After confirming the expression of mTOR, TSC1 and TSC2 following indicated treatments (Figure S1B), we found that miR-7-5p was more 
enriched in exosomes than in intracellular when Everolimus was employed. On the contrary, miR-7-5p located in intracellular rather than in exosomes when siTSC1/2 was performed. When siTSC1/2-A549 cells were treated by Everolimus, miR-7-5p would be enriched in exosomes again (Fig. 1H). It confirmed that the activity of mTORC1 controlled the redistribution of miR-7-5p.

Rab27A and Rab27B are two closely related Rab small GTPases, which play key roles in the secretion of exosomes in many types of cells [23]. The knockdown of Rab27A and Rab27B by siRNA would reduce exosome secretion of HeLa cells. And Rapamycin targeting mTORC1 stimulated the release of exosome dependent on Rab27A [17]. Here, we found that the release of miR-7-5p-loaded exosomes was significantly reduced to almost undetectable when Rab27A or Rab27B was knocked down (Figure S1C), accompanied by the enrichment of intracellular miR-7-5p. When Everolimus were performed in the cells after Rab27A or Rab27B knocking down, the exocytosis of miR-7-5p loaded exosomes was also suppressed (Fig. 1I). This revealed that the release of miR-7-5p-loaded exosomes induced by Everolimus depended on Rab27A and Rab27B.

\section{Loss of intracellular miR-7-5p induced phosphorylation of MNK/elF4E axis, but supplement of extra exosomal miR-7-5p could reverse it}

With the gradual deepening of understanding, it had been found that there were feedbacks of multiple kinases during the employment of Everolimus, such as hyperphosphorylation of elF4E at the Ser209 site, which led to the development of drug resistance[24]. The mTOR inhibitor Rapalog induced elF4E phosphorylation was done by kinases termed MAP kinase-interacting kinases (Mnks) independently of the conventional Mnk-activating MAPK pathway[25]. Coincidentally, MNK1 was a potential targeted gene of miR-7-5p by bioinformatics analysis (Pictar, TargetScan, and Tarbase) (Fig. 2A). It could directly bind to the 3'UTR of mRNA of MNK1 by the luciferase reporter gene test (Fig. 2B). When miR-7-5p was elevated by mimics, the MNK1 would be down-regulated. On the contrary, inhibition of miR-7-5p by inhibitor could upregulate the MNK1 as showing in the Fig. 2C and Figure S1D. NSCLC cell lines A549 and SPC-A1 were utilized to conduct the following experiments due to that miR-7-5p level was lower but MNK1 expression was higher compared with the human bronchial epithelial (HBE) cell and other NSCLC cell lines (Figure S1E). Therefore, a lentivirus-based system (LVmiR-7-5p and LV-NC) was used to conduct stable miR-7-5p overexpressed A549 and SPC-A1 cell lines. When stable miR7-5p A549 and SPC-A1 cells were treated with Everolimus, overexpressed miR-7-5p was able to inhibit upregulation of MNK1 and p-eIF4E $\mathrm{E}^{\mathrm{S} 209}$ induced by Everolimus without affection on the downstream of mTOR, including p-S6 $235 / 236$ and p-4EBP1 ${ }^{\text {Thr37/46 }}$ (Fig. 2D). Simultaneously, considering higher miR-7-5p can inhibit ubiquitin-mediated protein degradation [26], and MNK1 protein can be degraded by ubiquitination in addition to be regulated by phosphorylation[27], the stable miR-7-5p and control A549 cells were treated with protein synthesis inhibitor cycloheximide (CHX) to calculate the half-life of MNK1 protein. As show in Fig. 2E and 2F, the MNK1 protein decreased by 50\% within 7.785 hours in the control A549 cells, and 7.278 hours in the stable miR-7-5p A549 cells. The half-life of the MNK1 protein between the two groups was relatively close, which indicated that miR-7-5p had only few impacts on MNK1 protein stability. So, it could completely inhibit the activation of the MNK/elF4E axis induced by Everolimus. Hence, we tried to use miR-7-5p-loaded exosomes as a candidate therapy. Here, we used stable miR-7-5p overexpressed A549 cells derived exosomes. They were able to be internalized by the A549 cells (Fig. 2G), and the rate of internalization is greater than the rate of secretion induced by Everolimus (Fig. $2 \mathrm{H}$ ). The same as stable upregulation of miR-7-5p, they suppressed the phosphorylation of MNK/elF4E axis without activation of downstream of mTOR (Fig. 2I), which suggested that miR-7-5p-loaded exosomes derived from tumor cells might be served as a new mechanism to alleviate the Everolimus resistance in NSCLC.

\section{The decreased miR-7-5p and elevated MNK1 were associated with poor prognosis of NSCLC}


To investigate the clinical significance of miR-7-5p and MNK1 in NSCLC specimens, we firstly confirmed the lower levels of miR-7-5p in cancer tissues compared to paired adjacent tissues through qPCR (Fig. 3A), while the MNK1 levels significantly elevated in cancer tissues rather than paired adjacent tissues (Fig. 3B) in 34 cases of pairs fresh tissues. There was a negative correlation between the mRNA levels of miR-7-5p and MNK1 in NSCLC (Fig. 3C, $R=-0.3004, P=$ 0.0128). Then, we further detected miR-7-5p by in-situ hybridization and MNK1 protein by immunohistochemistry in 318 cases of paraffin-embedded NSCLC tissues (including 161 cases of lung adenocarcinoma and 157 cases of lung squamous cell carcinoma) and 90 cases of adjacent tissues respectively. Positive expression of miR-7-5p and MNK1 was mainly located in the cytoplasm (Fig. 3D). Strongly positive miR-7-5p was observed in normal alveolar epithelial cells, accompanied by weak staining of MNK1 protein expression. In adenocarcinoma and squamous cell carcinoma tissues, low expression of miR-7-5p was found, while elevated expression of MNK1 protein was indicated (Fig. 3E).

Univariate analysis showed that lower miR-7-5p was more prone to occur lymph node metastasis (LNM) $(P=0.025$, Table 1). And compared with the primary focus, the levels of miR-7-5p were lower in metastases (Fig. 3F). Besides, lower miR-7-5p $(P=0.048)$ or positive MNK1 $(P=0.007)$ had poor survival status (Table 1$)$. Higher miR-7-5p combined with negative MNK1 would behave as a better survival status $(P=0.004)$ (Table 1$)$. We also calculated overall survival (OS) for NSCLC patients with lower miR-7-5p, higher MNK1 and the combined index of miR-7-5p and MNK1 respectively (Fig. 3G). The NSCLC patients tended to have poor prognosis if miR-7-5p was lower (Fig. 3G, upper), or MNK1 was positive (Fig. 3G, middle). Optimal outcomes were observed in the expression patterns of combined higher miR-7-5p and negative MNK1. But lower miR-7-5p and positive MNK1 coexist with a herald of the worst prognosis. And the prognosis was intermediate in other phenotype with no significant statistical difference (Fig. 3G, lower). Multivariate regression analysis showed that besides LNM status, clinical stages, gender and pathological grades, miR-7-5p and MNK1 are also independent prognostic factors for NSCLC (Table 2). Correlation analysis indicated that there was a significant negative correlation between the miR-7-5p and MNK1 (Table 3, $r=-0.143, P=0.011$ ). The human NSCLC clinical data supported the concept that miR-7-5p appeared as a tumor suppressor, but MNK1 had an oncogenic function. The balance of the two biomarkers plays an important role in the pathogenesis of NSCLC. Therefore, restoring the miR-7-5p expression levels in the NSCLC cells could have a tumor suppressor effect. 
Table 1

Analysis of the association between expression of hsa-miR-7-5p and MNK1 and clinicopathological features of NSCLC $(n=318)$.

\begin{tabular}{|c|c|c|c|c|c|c|c|c|c|}
\hline \multirow{2}{*}{$\begin{array}{l}\text { Clinicopathological } \\
\text { features }(n)\end{array}$} & \multicolumn{3}{|l|}{ miR-7-5p } & \multicolumn{3}{|l|}{ MNK1 } & \multicolumn{3}{|c|}{ miR-7-5p/MNK1 ${ }^{\#}$} \\
\hline & Low (\%) & High (\%) & $\begin{array}{l}P \text { - } \\
\text { value }\end{array}$ & Po (\%) & $\begin{array}{l}\mathrm{Ne} \\
(\%)\end{array}$ & P-value & $\begin{array}{l}\mathrm{N}^{-} \\
\text {(\%) }\end{array}$ & $\begin{array}{l}\mathrm{P}^{+} \\
(\%)\end{array}$ & P-value \\
\hline
\end{tabular}

\section{Age (years)}

\begin{tabular}{|c|c|c|c|c|c|c|c|c|}
\hline$\leq 55(n=149)$ & $\begin{array}{l}73 \\
(49.0)\end{array}$ & $76(51.0)$ & 0.933 & $75(50.3)$ & $\begin{array}{l}74 \\
(49.7)\end{array}$ & 0.243 & $\begin{array}{l}40 \\
(26.8)\end{array}$ & $\begin{array}{l}109 \\
(73.2)\end{array}$ \\
\hline$>55(n=169)$ & $82(48.5)$ & 87 (51.5) & & $\begin{array}{l}74 \\
(43.8)\end{array}$ & $\begin{array}{l}95 \\
(56.2)\end{array}$ & & $\begin{array}{l}58 \\
(34.3)\end{array}$ & $\begin{array}{l}111 \\
(65.7)\end{array}$ \\
\hline
\end{tabular}

\section{Gender}

\begin{tabular}{|c|c|c|c|c|c|c|c|c|c|}
\hline Male $(n=241)$ & $\begin{array}{l}116 \\
(48.1)\end{array}$ & 125(51.9) & 0.701 & $\begin{array}{l}108 \\
(44.8)\end{array}$ & $\begin{array}{l}133 \\
(55.2)\end{array}$ & 0.197 & $\begin{array}{l}79 \\
(32.8)\end{array}$ & $\begin{array}{l}162 \\
(67.2)\end{array}$ & \\
\hline Female $(n=77)$ & $\begin{array}{l}39 \\
(50.6)\end{array}$ & $38(49.4)$ & & $\begin{array}{l}41 \\
(53.2)\end{array}$ & $\begin{array}{l}36 \\
(46.8)\end{array}$ & & $\begin{array}{l}19 \\
(24.7)\end{array}$ & $\begin{array}{l}58 \\
(75.3)\end{array}$ & \\
\hline
\end{tabular}

\section{LNM status}

\begin{tabular}{|c|c|c|c|c|c|c|c|c|}
\hline NO LNM $(n=131)$ & $54(41.2)$ & 77 (58.8) & $0.025^{\star}$ & $\begin{array}{l}56 \\
(42.7)\end{array}$ & $\begin{array}{l}75 \\
(57.3)\end{array}$ & 0.219 & $\begin{array}{l}47 \\
(35.9)\end{array}$ & $\begin{array}{l}84 \\
(64.1)\end{array}$ \\
\hline LNM $(n=187)$ & $\begin{array}{l}101 \\
(54.0)\end{array}$ & $86(46.0)$ & & $\begin{array}{l}93 \\
(49.7)\end{array}$ & $\begin{array}{l}94 \\
(50.3)\end{array}$ & & $\begin{array}{l}51 \\
(27.3)\end{array}$ & $\begin{array}{l}136 \\
(72.7)\end{array}$ \\
\hline
\end{tabular}

\section{Pathologic grades}

\begin{tabular}{|c|c|c|c|c|c|c|c|c|}
\hline $\begin{array}{l}\text { Well/Moderate }(\mathrm{n} \\
=145)\end{array}$ & $\begin{array}{l}71 \\
(49.0)\end{array}$ & $74(51.0)$ & 0.942 & $\begin{array}{l}74 \\
(51.0)\end{array}$ & $\begin{array}{l}71 \\
(49.0)\end{array}$ & 0.172 & $\begin{array}{l}42 \\
(29.0)\end{array}$ & $\begin{array}{l}103 \\
(71.0)\end{array}$ \\
\hline Poor $(n=173)$ & $\begin{array}{l}84 \\
(48.6)\end{array}$ & $89(51.4)$ & & $\begin{array}{l}75 \\
(43.4)\end{array}$ & $\begin{array}{l}98 \\
(56.6)\end{array}$ & & $\begin{array}{l}56 \\
(32.4)\end{array}$ & $\begin{array}{l}117 \\
(67.6)\end{array}$ \\
\hline
\end{tabular}

\section{Clinical stages}

\begin{tabular}{|c|c|c|c|c|c|c|c|c|c|}
\hline $\begin{array}{l}\text { Stage I and II }(\mathrm{n}= \\
166)\end{array}$ & $\begin{array}{l}76 \\
(45.8)\end{array}$ & $90(54.2)$ & 0.270 & $\begin{array}{l}77 \\
(46.4)\end{array}$ & $\begin{array}{l}89 \\
(53.6)\end{array}$ & 0.861 & $\begin{array}{l}56 \\
(33.7)\end{array}$ & $\begin{array}{l}110 \\
(66.3)\end{array}$ & 0.239 \\
\hline Stage $\nabla(n=152)$ & $\begin{array}{l}79 \\
(52.0)\end{array}$ & $73(48.0)$ & & $\begin{array}{l}72 \\
(47.4)\end{array}$ & $\begin{array}{l}80 \\
(52.6)\end{array}$ & & $\begin{array}{l}42 \\
(27.6)\end{array}$ & $\begin{array}{l}110 \\
(72.4)\end{array}$ & \\
\hline
\end{tabular}

\section{Survival status}

\begin{tabular}{|c|c|c|c|c|c|c|c|c|c|}
\hline Alive $(n=210)$ & $94(44.8)$ & $116(55.2)$ & $0.048 *$ & $\begin{array}{l}87 \\
(41.4)\end{array}$ & $\begin{array}{l}123 \\
(58.6)\end{array}$ & $0.007 * *$ & $\begin{array}{l}76 \\
(36.2)\end{array}$ & $\begin{array}{l}134 \\
(63.8)\end{array}$ & $0.004^{\star \star}$ \\
\hline Dead $(n=108)$ & $\begin{array}{l}61 \\
(56.5)\end{array}$ & 47 (43.5) & & $\begin{array}{l}62 \\
(57.4)\end{array}$ & $\begin{array}{l}46 \\
(42.6)\end{array}$ & & $\begin{array}{l}22 \\
(20.4)\end{array}$ & $\begin{array}{l}86 \\
(79.6)\end{array}$ & \\
\hline
\end{tabular}

${ }^{*}$ Chi-square test, statistically significant difference $\left({ }^{\star} P<0.05,{ }^{\star *} P<0.01\right)$.

Abbreviations: ADC, adenocarcinoma; $\mathrm{H}$, High expression; L, Low expression; Po, Positive expression; Ne, Negative expression; LNM, lymph node metastasis; NSCLC: non-small cell lung cancer; SCC, squamous cell carcinoma; miR-7$5 p / M N K 1^{\#}, N^{-}$, the higher miR-7-5p combined with negative expression of MNK1,

$\mathrm{P}^{+}$, other combination of expression of these two factors. 
Table 2

Summary of multivariate of Cox proportional regression for overall survival in 318 cases of NSCLC.

\begin{tabular}{|c|c|c|c|c|c|c|c|}
\hline \multirow[t]{2}{*}{ Parameter } & \multirow[t]{2}{*}{ B } & \multirow[t]{2}{*}{ SE } & \multirow[t]{2}{*}{ Wald } & \multirow[t]{2}{*}{ Sig. } & \multirow[t]{2}{*}{$\operatorname{Exp}(B)$} & \multicolumn{2}{|c|}{$95.0 \% \mathrm{Cl}$ for $\operatorname{Exp}(\mathrm{B})$} \\
\hline & & & & & & Lower & upper \\
\hline Age & -.153 & .199 & .593 & .441 & .858 & .580 & 1.268 \\
\hline Gender & -.521 & .249 & 4.357 & $.037 *$ & .594 & .364 & .969 \\
\hline Histological types & -.040 & .215 & .034 & .853 & .961 & .630 & 1.465 \\
\hline LNM status & -.976 & .252 & 15.020 & $.000 * \star \star$ & .377 & .230 & .617 \\
\hline Clinical stages & -.634 & .216 & 8.632 & $.003^{\star \star \star}$ & .530 & .347 & .810 \\
\hline Pathological grades & -.486 & .202 & 5.801 & $.016^{\star}$ & .615 & .414 & .913 \\
\hline miR-7-5p & .394 & .199 & 4.007 & $.045^{\star}$ & 1.491 & 1.008 & 2.204 \\
\hline MNK1 & -.464 & .206 & 5.095 & $.024^{\star}$ & .629 & .420 & .941 \\
\hline
\end{tabular}

Table 3

The pairwise association between expression of miR-7-5p and MNK1 protein in 318 cases of NSCLC

\begin{tabular}{|llll|}
\hline \multicolumn{4}{|c|}{ MNK1 } \\
\hline & Positive (\%) & Negative (\%) & $P$-value \\
\hline miR-7-5p & & \\
\hline High (\%) & $65(39.9)$ & $98(60.1)$ & $0.011^{\star}$ \\
\hline Low (\%) & $84(54.2)$ & $71(45.8)$ & $(r=-0.143)$ \\
\hline *Spearman's rank correlation test, statistically significant difference $\left({ }^{*} P<0.05\right)$ \\
\hline
\end{tabular}

Exosomal miR-7-5p enhanced the anticancer effect of Everolimus in vitro.

Exogenous miR-7-5p could supply the deficiency of it in cells. Further contrast among the groups of Everolimus, exogenous miR-7-5p and combined of them, we discovered that Everolimus alone or stable miR-7-5p expression could inhibit NSCLC cell proliferation (Fig. 4A \& S2A), clone formation (Fig. 4B \& S2B) and 2D\&3D migration capabilities (Fig. 4C-4D \& S2C-S2D, respectively). When Everolimus was carried out to treat the stable miR-7-5p cells, the inhibitory effect was the most significant.

As the functions of exosomes mediated by their contents are more widely recognized, they have been proposed as a potential cell-based alternative therapy which have received increasing attention[28]. Due to the direct sorting and packaging of nucleic acids into exosomes may not provide the functionally active contents into recipient cells effectively[29]. We tried to use exosomes derived from tumor cells directly to improve miR-7-5p in Everolimus treated NSCLC cells. Like exogenous miR-7-5p, exosomal miR-7-5p could inhibit the cell proliferation (Fig. 4E \& S2E), clone formation (Fig. 4F \& S2F) and 2D\&3D migration capabilities (Fig. 4G-4H \& S2G-S2H, respectively). And further combination of miR-7-5p-loaded exosomes with Everolimus showed a more obvious tumor suppressive effect, which 
indicated that exo-miR-7-5p could be internalized by NSCLC cells and cooperated with Everolimus to enhance the anticancer effect of it in vitro.

\section{Exosomal miR-7-5p enhanced the anticancer therapeutic efficacy of Everolimus in vivo.}

We used LV-miR-7-5p and LV-NC A549 cells to construct nude mouse subcutaneous tumor models respectively. Once the tumor volume reached $50-100 \mathrm{~mm}^{3}$, nude mouses would be divided into four groups and received indicated treatments. The growth rate of the LV-miR-7-5p group was significantly slower than the control group, as well as the tumor volume. It was worth noting that although Everolimus had a strong inhibitory effect on tumor growth in the early stage, its growth rate tended to accelerate in the later period, resulting in the relatively bigger volume than LV-miR-7-5p combined Everolimus group. The proliferation rate of tumor cells and tumor volume always kept at a lowest level when LV-miR-7$5 p$ was performed with Everolimus (Fig. 5A-5D). Further, in spite of that Everolimus could suppress the Ki-67 index of tumors than the control groups no matter combined with LV-NC or LV-miR-7-5p, the activation of MNK1/elF4E axis was only observed in the group of LV-NC combined Everolimus (Fig. 5E, Figure S3A), meant restoring the levels of miR-7-5p could be used as an effective method to reverse the activation of MNK/elF4E axis induced by Everolimus.

Currently, the treatment of cancers highlights the need for more effective and innovative therapies. Because of safer biological behaviors, exosomes have the value of translational medicine for clinical treatment purposes[29]. Using tumor-derived exosomes will provide additional competitive advantages for the selective delivery of anticancer therapies not only to the primary tumor but also to the premetastatic niche and even to metastasis, owing to their intrinsic organotropic tumor-homing properties[30]. We further established the abdominal metastasis models accepting the indicated treatments to monitor tumors in vivo and the exosomal miR-7-5p in the blood. Tumor involvement was observed in all groups (Fig. 5F and Figure S3B). Notably, the exo-miR-7-5p combined with Everolimus had the weakest fluorescence signal (Fig. 5G), and high miR-7-5p in the blood (Fig. 5H).

\section{Combination of miR-7-5p with Everolimus induced apoptosis to exhibit a synergistic anticancer therapeutic efficacy via dual abrogation of MNKJelF4E and mTOR in NSCLC}

The function of elF4E is relay on the subcellular locations of it. In the initiation phase of translation, most mRNAs are controlled by intracytoplasmic elF4E. And if it locates in the nucleus, the export of certain mRNAs to the cytoplasm will depend on it [31]. In order to further explore the specific value and mechanism of the combined targeting of the MNK/elF4E axis and the mTOR pathway in the treatment of NSCLC, the first synthetic small molecule MNK1 inhibitor CGP57380 was performed to block the MNK/elF4E axis. We found p-MNK1 Thr197/202 was mainly distributed in the nucleus and was inhibited. In spite of p-elF4E $\mathrm{E}^{\mathrm{S} 209}$ was located both in the cytoplasm and nucleus, CGP57380 mainly suppressed the phosphorylation of intracytoplasmic elF4E (Fig. 6A, Figure S4A). Besides, it still has characteristics such as poor specificity and relatively weak affinity, which limits its value in clinical treatment [32]. Fortunately, both endogenous and exosomal miR-7-5p could restore the intracellular tumor suppressor miR-7-5p and reverse the feedback activation of MNK/elF4E axis more specific and safer. When stable LV-miR-7-5p A549 and SPC-A1 cells were treated by Everolimus (Fig. 6B, Figure S4B) or combination of Everolimus with the miR-7-5p-loaded exosomes derived from A549 cells (Fig. 6C, Figure S4C), the activation of the MNK/elF4E axis was eliminated.

Further flow cytometry analysis indicated the apoptosis rate of miR-7-5p mimics or Everolimus treatment alone was less than the combined group (Fig. 6D, Figure S4D). And representative microscopic images of liver metastatic lesions among all groups in previous abdominal metastasis models observed obvious necrosis of metastases in exo-miR-7-5p combined with Everolimus group (Fig. 6E), which suggested that the combination of miR-7-5p with Everolimus could significantly strengthen the anticancer effect of Everolimus through inducing cell apoptosis. Caspase-3, cleaved caspase-3, DR4, DR5, pro-apoptotic proteins Bak, Bad and Bax were significantly up-regulated but anti-apoptotic protein 
Bcl-xL was down-regulated (Fig. 6F-H, Figure S4E-G), when the stable LV-miR-7-5p NSCLC cells were treated with Everolimus, results indicated that it could induce the co-activation of the inherent mitochondrial apoptosis and the death receptor pathway.

\section{Discussion}

In NSCLC, the PI3K-AKT-mTOR pathway activation has been implicated with EGFR TKIs, which represents $14.9 \%$ in EGFR TKI resistance events[5]. Everolimus is a derivative of rapamycin, which could inhibit the activity of mTORC1 and might be a potential therapeutic drug in NSCLC. Regrettably, the single drug activity in the cancers is limited[33]. Multiple studies have shown that targeted inhibition of mTOR is able to activate a variety of survival signaling pathways, including PI3K/Akt, MEK/ERK and MNK/elF4E, which will provide cancer cells with a survival advantage and ultimately blunting rapamycin treatment[24]. In addition, long-term effects of rapamycin lead to changes of intracellular miRNA expression profile. In rapamycin-resistant cells, oncogenic miRNAs were significantly up-regulated, while tumor suppressor miRNAs were down-regulated. The tumor suppressor miRNA-101 in the cell culture medium of two clear cell renal cell carcinoma cell lines increased by the usage of mTOR inhibitors, indicating that mTOR inhibitors promoted the excretion of it into the cellular microenvironment to neutralize its anti-tumor activity[34]. After comparing the expression profiles of miRNA between parental myoblasts and drug-resistant strains, there was down-regulation of miR-7a[9]. This is consistent with our research. We confirmed that Everolimus promoted the decreasing of miR-7-5p in NSCLC. The bioinformatics predictions and luciferase experiments validated that miR-7-5p could target the mRNA of MNK1. As the elF4E phosphorylation was increased by mTOR inhibitors in a MNK-dependent manner[35]. We speculated that Everolimus-mediated activation of MNK/elF4E might be related to miR-7-5p, which had not been studied before. So, we elevated or suppressed the miR-7-5p by mimics or inhibitor, and combined with Everolimus or not to further test the phosphorylation of MNK/elF4E axis and the activity of downstream molecules of mTOR pathway in NSCLC cells. It was confirmed that up-regulation of miR-7-5p could indeed inhibit the phosphorylation of the MNK/elF4E axis without feedback activating of mTOR pathway. However, miR-7-5p could inhibit protein ubiquitinated degradation[26]. And MNK1 protein could be degraded by ubiquitination in addition to being regulated by phosphorylation[27]. If the upregulation of miR-7-5p inhibits the ubiquitinated degradation of MNK1, it would offset the tumor suppressor effect of miR-7-5p. Hence, we further verified that the targeted inhibitory effect of miR-7-5p on MNK protein was independent on the proteasome mediated degradation of MNK1. The above studies indicated that Everolimus targeted mTORC1 inducing the loss of intracellular miR-7-5p to activate MNK/elF4E axis mediating Everolimus resistance. This has not been reported in other studies, and it is worthy of further research and exploration.

In recent years, many studies have shown that miR-7 may be a tumor suppressor, and are significantly downregulated. But there were also some opposite reports[13]. In NSCLC, the role of miR-7 was also contradictory. It was found that EGFR promotes lung tumorigenesis by activating miR-7[36]. While miR-7 was significantly higher in serum exosomes from healthy controls than from patients with lung carcinoma, and higher miR-7 was associated with strong response to lung carcinoma patients receiving gefitinib treatment[37]. Therefore, further research is essential to identify whether miR7 is a oncomiR or tumor suppressor in NSCLC. Here, we found that there was lower expression of miR-7-5p in multiple NSCLC cell lines, fresh tissues and paraffin-embedded tissues of NSCLC, which was related to the poor prognosis of patients. When miR-7-5p was overexpressed by lentivirus or exosomes, it could inhibit the proliferation, growth and migration of NSCLC in vivo and in vitro. Our research supported that miR-7-5p had tumor suppressor value in NSCLC. As for the apparently contradictory functions of miR-7-5p, it might be explained by the balance of the positive or negative feedback loops in different cancer types or stages.

The transfer of miRNAs by exosomes is considered to be a novel and important mechanism of genetic exchange between cells[38]. Through analyzing TCGA database, it was found that if the mTORC1 was low, the extracellular exosomes would be significantly enriched. Exactly, miR-7-5p was recruited in exosomes, which could be inhibited by the

Page $13 / 26$ 
exosome inhibitor GW4869. Hence, we speculated Everolimus could stimulate NSCLC cells to excrete miR-7-5p-loaded exosomes, thereby reducing intracellular miR-7-5p to weaken the anti-tumor activity of Everolimus. Previous studies believed that mTORC1 controlled cell metabolism by removing organelles and components, which was controlled by the main mechanism of autophagy. Recently, studies have found that the release of exosomes and autophagy were two related processes leading to the loss of cell membrane and diverse contents. The release of exosomes was also regulated by $\mathrm{mTORC1}$ [17]. Everolimus suppressed the activity of mTORC1 inducing the release of exosomes. In contrast the release of exosomes was significantly reduced after activation of mTORC1 by knockdown of TSC1/2, important negative regulators of $\mathrm{mTORC1}$. Besides, the regulation of release of exosomes by MTORC1 was also depended on two crucial exosomes regulatory proteins, Rab27A and Rab27B. They might participate the fusion of vesicles and plasma membrane in the process of exosomes secretion [39]. Different from other research, Everolimus induced exosomes derived from NSCLC cells were showed up as enrichment of miR-7-5p. There might be a mechanism involved in the sorting process of miR-7-5p into exosomes derived from Everolimus treated NSCLC cells. According to the report, the motif of "GUUG" was associated with indicated miRNAs into exosomes in SW620 cell[40]. Coincidentally, the base sequence of miR-7-5p (UGGAAGACUAGUGAUUUUUGUUGU) contains the "GUUG" motif, which might be a candidate reason for miR-7-5p sorting process. But the possible mechanism needs further verification.

The abnormal miRNA profile of tumor cells with Everolimus treatment also supported corresponding targeted therapy strategies. And therapeutic intervention might be aimed at restoring normal miRNA levels[41]. Therefore, restoration of miR-7-5p could sensitize the anti-cancer effect of Everolimus. The exosomes could be used as carriers, which had the value of low immunogenicity, low toxicity, and high stability via being modified to improve loading and targeting efficiency[42]. The miRNAs loaded in exosomes relied on the transcripts of parental cells [43]. Over-expression of miRNAs in parental cells will increase indicated miRNA in extracellular vesicles (EVs) [44]. Therefore, the efflux exosomes derived from miR-7-5p overexpressed NSCLC cells had a higher content of miR-7-5p, which could be internalized by A549 cells. It was further confirmed in vitro and in vivo that miR-7-5p-loaded exosomes derived from A549 cells could inhibit the proliferation, clone formation, and migration capabilities of NSCLC cells, indicating that exosomes would have an important potential value in tumor treatment. This was consistent with the previous preclinical studies. Breast cancer (MDA-MB-231) cell-derived exosomes (231-Exo) could be specifically internalized by NSCLC, and miRNA-126 loaded 231Exo (MDA-MB-231 cell-derived exosomes) strongly suppressed A549 lung cancer cell proliferation and migration[45]. And after transfecting with a plasmid contained miRNA-122, EVs were isolated from stromal cell culture medium derived from adipose tissue to induce hepatocytes for sensitizing to sorafenib [46]. Although there was no definitive result at present, some clinical trials were ongoing to evaluate the efficacy of miRNA and exosomes as therapeutic agents[47]. The study (https://clinicaltrials.gov: NCT02507583) aimed to activate the immune system through exosomes released by glioblastoma cells. Compared with traditional therapies, activation of the immune system by glioblastoma cells derived exosomes in patients with glioma are able to reduce risks and increase benefits. Therefore, based on the changes of miRNA profile in NSCLC cells induced by Everolimus, here we used tumor cell-derived exosomes to restore miR-7-5p in cells, and combined treatment with Everolimus, which has an important clinical application value.

The compensation feedback mechanism links the AKT/mTOR pathway with the MNK/elF4E axis. The downregulation of one pathway was associated with the activation of another one, which subsequently supported cell proliferation and cancer survival[48]. The complexity of cell signaling means that traditional single treatment methods were usually ineffective, further multiple targets were needed to be found [49]. The efficacy of combined targeted therapy was significantly better than that of single medication. The MNK deletion can reduce basal mTORC1 signaling and MNK activation contributes to rapamycin resistance in cancer cells by sustaining mTORC1 activity following rapamycin treatment[50]. The combination of MNK inhibitor CGP57380 with Everolimus overcomes Everolimus-mediated elF4E phosphorylation and shows enhanced cytotoxicity against T-cell acute lymphoblastic leukemia cells[48]. However, since the discovery of CGP57380 in low micromolar concentration with MNK inhibitory effect, little progress was found in

Page $14 / 26$ 
clinical treatment. Currently, the development of Mnk inhibitors for the treatment of disease is still below expectation[51]. It is urgent to find new effective methods that can target the MNK/elF4E axis. The ability to regulate miRNA levels and activity in vivo by miRNA analogs or anti-miRNAs was to develop innovative cancer treatment strategies. To supplement the down-regulated tumor suppressor miRNA-7-5p induced by Everolimus could target the expression of MNK1 protein, thereby inhibiting the feedback activation of the MNK/elF4E axis, sensitizing the Everolimus and achieving the combined inhibition of mTOR and MNK/elF4E pathway in NSCLC. Here, we found that when miR-7-5p loaded exosomes derived from LV-miR-7-5p A549 cells combined with Everolimus for dual targeting the MNK/elF4E axis and mTOR pathway, could induce apoptosis of NSCLC cells through the mitochondrial pathway and the death receptor pathway. It was more effective than our previous usage of CGP57380 in combination with Everolimus in NSCLC, which only promoted apoptosis through the mitochondrial pathway. It was reported that enforced levels of exogenous miR-7 in TRAILoverexpressed MSCs might sensitize the treatment of TRAIL to increase apoptosis in the death receptor pathway via targeting XIAP in glioma cells, meanwhile, the level of miR-7 was positively correlated with apoptosis [52]. We speculated the up-regulation of miR-7-5p was able to inhibit other targets which associated with death receptor pathway-mediated apoptosis. This apoptosis-based cancer treatment strategy could selectively activate death-induced molecules in the apoptosis program of transformed cells for tumor eradication, which provided important support for the clinical application of tumor suppressor miR-7-5p.

\section{Conclusions}

Everolimus stimulates the release of miR-7-5p loaded exosomes from NSCLC cells in Rab27A and Rab27B dependent manners, thereby reducing the intracellular miR-7-5p levels, which attenuates the inhibition of MNK1 and subsequently promotes MNK-dependent elF4E phosphorylation to blunt effectiveness of itself in NSCLC. Both endogenous and exosomal miR-7-5p all have the synergy with Everolimus, which not only inhibits the proliferation, migration, and metastasis of NSCLC in vitro and in vivo, but also promotes the apoptosis of NSCLC through dual targeting MNK/elF4E axis and mTOR pathway (Fig. 6l). Besides, both low level of miR-7-5p and positive expression of MNK1 can act as independent poor prognostic biomarkers for NSCLC. Therefore, exosome-mediated miR-7-5p delivery combined with Everolimus may be considered as a promising novel combined therapeutic strategy for NSCLC.

\section{Abbreviations}

NSCLC: non-small cell lung cancer; MNK: mitogen-activated protein kinase interacting kinases; elF4E: eukaryotic translation initiation factor 4E; mTOR: mammalian target of rapamycin; mTORC1: mTOR complex 1; mTORC2: mTOR complex 2; p70S6K: 70 kDa ribosomal protein S6 kinase; TMA: tissue microarrays; Non-CLT: non-cancerous lung tissue; FSB: fetal bovine serum; H\&E: hematoxylin and eosin; IHC: Immunohistochemistry; ISH: In-situ hybridization; qPCR: Quantitative PCR; OS: Overall survival; LNM: lymph node metastasis; TEM: transmission electron microscope; TSC1: Tuberous sclerosis complex 1; TSC2: Tuberous sclerosis complex 2; Rab27: RAB27A member RAS oncofamily; Rab27B: RAB27B member RAS oncofamily; PBS: phosphate buffered saline.

\section{Declarations}

\section{Acknowledgements}

We thank all researchers in Cancer Research Institute, School of Basic Medical Sciences, Central South University with experimental assistance.

\section{Author's contributions}

Page 15/26 
Songqing Fan, Weiyuan Wang and Jian Ma were involved in the conception and design of the study. Sile Liu, Yue Ning, Hongmei Zheng, Yuting Zhan, Haihua Wang, Yang yang, Jiadi Luo, Qiuyuan Wen, Hongjing Zang and Jinwu Peng were involved in the analysis and interpretation of the data. Weiyuan Wang, Sile Liu and Songqing Fan were involved in the writing, reviewing and/or revision of the manuscript. All authors have read and approved the final manuscript.

\section{Funding}

This study was supported by the National Nature Science Foundation of China (No. 81773218,81972838 and 81802791).

\section{Availability of data and materials}

The datasets used and/or analyzed during the current study are available from the corresponding author on reasonable request.

\section{Ethics approval and consent to participate}

Protocols were approved by the Second Xiangya Hospital of Central South University Ethics Review Board (Scientific and Research Ethics Committee, No. K021/2021). All patients involved in our study had written informed consent.

\section{Consent for publication}

Not applicable.

\section{Competing interests}

The authors declare that they have no competing interests.

\section{Supplemental information}

We describe antibodies, reagents and cells in details in the Supplemental Material and Methods.

\section{References}

1. Tan AC. Targeting the PI3K/Akt/mTOR pathway in non-small cell lung cancer (NSCLC). Thoracic Cancer. 2020; 11: $511-8$

2. Guertin DA, Sabatini DM. Defining the role of mTOR in cancer. Cancer Cell. 2007; 12: 9-22.

3. Kong N, Tao W, Ling X, Wang J, Xiao Y, Shi S, et al. Synthetic mRNA nanoparticle-mediated restoration of p53 tumor suppressor sensitizes p53-deficient cancers to mTOR inhibition. Science Translational Medicine. 2019; 11:eaaw1565.

4. Dong S, Zhang XC, Cheng H, Zhu JQ, Chen ZH, Zhang YF, et al. Everolimus synergizes with gefitinib in non-small-cell lung cancer cell lines resistant to epidermal growth factor receptor tyrosine kinase inhibitors. Cancer Chemotherapy \& Pharmacology. 2012; 70: 707-16.

5. Fang W, Huang Y, Gu W, Gan J, Zhang L. PI3K-AKT-mTOR pathway alterations in advanced NSCLC patients after progression on EGFR-TKI and clinical response to EGFR-TKI plus everolimus combination therapy. Translational Lung Cancer Research. 2020; 9: 1258-67.

6. Justin S, Rutz J, Maxeiner S, Chun KH, Blaheta RA. Bladder Cancer Metastasis Induced by Chronic Everolimus Application Can Be Counteracted by Sulforaphane In Vitro. International Journal of Molecular Sciences. 2020; 21 : 5582. 
7. Katsha A, Wang L, Arras J, Omar OM, El-Rifai W. Activation of ElF4E by Aurora Kinase A Depicts a Novel Druggable Axis in Everolimus-Resistant Cancer Cells. Clinical Cancer Research An Official Journal of the American Association for Cancer Research. 2017; 23: 3756-68.

8. Yoshizawa A, Fukuoka J, Shimizu S, Shilo K, Franks TJ, Hewitt SM, et al. Overexpression of phospho-elF4E is associated with survival through AKT pathway in non-small cell lung cancer. Clinical Cancer Research. 2010; 16: $240-8$

9. Totary-Jain H, Sanoudou D, Ben-Dov IZ, Dautriche CN, Guarnieri P, Marx SO, et al. Reprogramming of the MicroRNA Transcriptome Mediates Resistance to Rapamycin. Journal of Biological Chemistry. 2013; 12: 861-2.

10. Qiuyuan, Wen, Weiyuan, Wang, Jiadi, Luo, et al. CGP57380 enhances efficacy of RAD001 in non-small cell lung cancer through abrogating mTOR inhibition-induced phosphorylation of elF4E and activating mitochondrial apoptotic pathway. Oncotarget. 2016;7: 27787-801.

11. Liu S, Zang H, Zheng H, Wang W, Fan S. miR-4634 augments the anti-tumor effects of RAD001 and associates well with clinical prognosis of non-small cell lung cancer. Scientific Reports. 2020; 10:13079.

12. Xiaofei Z, Danfen Y, Yaqiang W. Overexpressed CDR1as functions as an oncogene to promote the tumor progression via miR-7 in non-small-cell lung cancer. Oncotargets \& Therapy. 2018; 11: 3979-87.

13. Kora P, Antica M, Matuli M. MiR-7 in Cancer Development. Biomedicines. 2021; 9: 325.

14. Webster RJ, Giles KM, Price KJ, Zhang PM, Mattick JS, Leedman PJ. Regulation of Epidermal Growth Factor Receptor Signaling in Human Cancer Cells by MicroRNA-7. Journal of Biological Chemistry. 2009; 284: 5731-41.

15. Wang Y, Liu J, Liu C, Naji A, Stoffers DA. MicroRNA-7 Regulates the mTOR Pathway and Proliferation in Adult Pancreatic $\beta$-Cells. Diabetes. 2013; 62: 887-95.

16. Wan MH, Ning B, Spiegel S, Lyon CJ, Hu TY. Tumor-derived exosomes (TDEs): How to avoid the sting in the tail. Medicinal Research Reviews. 2020;40:385-412.

17. Zou W, Lai M, Zhang Y, Zheng L, Bai X. Exosome Release Is Regulated by mTORC1. Advanced Science. 2018; 6:1801313.

18. Chu S, Wen Q, Qing Z, Luo J, Wang W, Chen L, et al. High expression of heat shock protein 10 correlates negatively with estrogen/progesterone receptor status and predicts poor prognosis in invasive ductal breast carcinoma. Human Pathology. 2017; 61: 173-80.

19. Fan S, Li Y, Yue P, Khuri FR, Sun SY. The elF4E/elF4G Interaction Inhibitor 4EGI-1 Augments TRAIL-Mediated Apoptosis through c-FLIP Down-regulation and DR5 Induction Independent of Inhibition of Cap-Dependent Protein Translation. Neoplasia. 2010; 12: 346-56.

20. Zhang X, Sai B, Wang F, Wang L, Wang Y, Zheng L, et al. Hypoxic BMSC-derived exosomal miRNAs promote metastasis of lung cancer cells via STAT3-induced EMT. Molecular Cancer. 2018;11:3979-87.

21. Qiuyuan, Wen, Weiyuan, Wang, Shuzhou, Chu, et al. Flot-2 Expression Correlates with EGFR Levels and Poor Prognosis in Surgically Resected Non-Small Cell Lung Cancer. PloS one. 2015;10:e0132190.

22. Liu W, Yi Y, Zhang C, Zhou B, Liao L, Liu W, et al. The Expression of TRIM6 Activates the mTORC1 Pathway by Regulating the Ubiquitination of TSC1-TSC2 to Promote Renal Fibrosis. Frontiers in Cell and Developmental Biology. 2021;8:616747.

23. Alexander M, Ramstead AG, Bauer KM, Lee SH, Runtsch MC, Wallace J, et al. Rab27-Dependent Exosome Production Inhibits Chronic Inflammation and Enables Acute Responses to Inflammatory Stimuli. Journal of Immunology. 2017; 199:3559-70.

24. Sun SY. mTOR-targeted cancer therapy: great target but disappointing clinical outcomes, why? Frontiers of Medicine. 2020;15:221-31. 
25. Fan C, Zhao C, Zhang F, Kesarwani M, Tu Z, Cai X, et al. Adaptive responses to mTOR gene targeting in hematopoietic stem cells reveal a proliferative mechanism evasive to mTOR inhibition. Proceedings of the National Academy of Sciences. 2020;118:e2020102118.

26. Sabater-Arcis M, Bargiela A, Furling D, Artero R. miR-7 Restores Phenotypes in Myotonic Dystrophy Muscle Cells by Repressing Hyperactivated Autophagy. Molecular Therapy Nucleic Acids. 2019;19:278-92.

27. Donovan KA, Ferguson FM, Bushman JW, Eleuteri NA, Fischer ES. Mapping the Degradable Kinome Provides a Resource for Expedited Degrader Development. Cell. 2020; 183: 1714-31.e10.

28. Kalluri R, LeBleu VS. The biology, function, and biomedical applications of exosomes. Science. 2020; 367:eaau6977.

29. Wei H, Chen Q, Lin L, Sha C, Zhu X. Regulation of exosome production and cargo sorting. International Journal of Biological Sciences. 2021; 17: 163-77.

30. Tumour exosome integrins determine organotropic metastasis. Nature. 2015; 527: 329-35.

31. Witzig TE, Reeder C, Han JJ, Laplant B, Stenson M, Tun HW, et al. The mTORC1 inhibitor everolimus has antitumor activity in vitro and produces tumor responses in patients with relapsed T-cell lymphoma. Blood. 2015; 126: 328-35.

32. Dreas A, Mikulski M, Milik M, Fabritius CH, Brzózka K, Rzymski T. Mitogen-activated Protein Kinase (MAPK) Interacting Kinases 1 and 2 (MNK1 and MNK2) as Targets for Cancer Therapy: Recent Progress in the Development of MNK Inhibitors. Current Medicinal Chemistry. 2017; 24:3025-53.

33. Lu X, Paliogiannis P, Calvisi D, Chen X. Role of the Mammalian Target of Rapamycin Pathway in Liver Cancer: From Molecular Genetics to Targeted Therapies. Hepatology. 2020; 73.

34. Nogueira I, Dias F, Morais M, Teixeira AL, Medeiros R. Everolimus resistance in clear cell renal cell carcinoma: miRNA-101 and HIF-2a as molecular triggers? Future Oncology. 2019; 15 Suppl 1:49-61.

35. Wang X, Yue P, Chan CB, Ye K, Ueda T, Watanabe-Fukunaga R, et al. Inhibition of Mammalian Target of Rapamycin Induces Phosphatidylinositol 3-Kinase-Dependent and Mnk-Mediated Eukaryotic Translation Initiation Factor 4E Phosphorylation. Molecular and Cellular Biology. 2007; 27: 7405-13.

36. Chou Y, Lin H, Lien Y, Wang Y, Hong C-F, Kao Y-R, et al. EGFR promotes lung tumorigenesis by activating miR-7 through a Ras/ERK/Myc pathway that targets the Ets2 transcriptional repressor ERF. Cancer research. 2010; 70 21: 8822-31.

37. Chen R, Qian Z, Xu X, Zhang C, Niu Y, Wang Z, et al. Exosomes-transmitted miR-7 reverses gefitinib resistance by targeting YAP in non-small-cell lung cancer. Pharmacological research. 2021; 65: 105442.

38. Cheng W, Wang K, Zhao Z, Mao Q, Wang G, Li Q, et al. Exosomes-mediated Transfer of miR-125a/b in Cell-to-cell Communication: A Novel Mechanism of Genetic Exchange in the Intestinal Microenvironment. Theranostics. 2020; 10: 7561 - 80.

39. Ostrowski M, Carmo NB, Krumeich S, Fanget I, Raposo G, Savina A, et al. Rab27a and Rab27b control different steps of the exosome secretion pathway. Nature Cell Biology. 2010; 12: 19-30; sup pp 1-13.

40. Gao T, Shu J, Cui J. A systematic approach to RNA-associated motif discovery. Bmc Genomics. 2018; $19: 146$.

41. Gorur A, Bayraktar R, Ivan C, Mokhlis HA, Bayraktar E, Kahraman N, et al. ncRNA therapy with miRNA-22-3p suppresses the growth of triple-negative breast cancer. Molecular Therapy Nucleic Acids. 2021; 23: 930 - 43.

42. Pi Y-N, Xia B, Jin M, Jin W-L, Lou G. Exosomes: powerful weapon for cancer nano-immunoengineering. Biochemical pharmacology. 2021;186: 114487.

43. Jian, Zhang, Sha, Li, Lu, Li, et al. Exosome and Exosomal MicroRNA: Trafficking, Sorting, and Function. Genomics Proteomics \& Bioinformatics. 2015;13(1):17-24.

44. Martellucci S, Orefice NS, Angelucci A, Luce A, Zappavigna S. Extracellular Vesicles: New Endogenous Shuttles for miRNAs in Cancer Diagnosis and Therapy? International Journal of Molecular Sciences. 2020; 21 :6486.

Page $18 / 26$ 
45. Nie H, Xie X, Zhang D, Zhou Y, Li B, Li F, et al. Use of lung-specific exosomes for miRNA-126 delivery in non-small cell lung cancer. Nanoscale. 2019;12:877-87.

46. Lou G, Song X, Yang F, Wu S, Wang J, Chen Z, et al. Exosomes derived from miR-122-modified adipose tissuederived MSCs increase chemosensitivity of hepatocellular carcinoma. Journal of Hematology \& Oncology. 2015; 8: 122.

47. Mohammed HR, Emine B, Gouda KH, Mohamed AE, Paola A, Arturo CR, et al. Exosomes: From Garbage Bins to Promising Therapeutic Targets. International Journal of Molecular Sciences. 2017; 18: 538.

48. Huang X, Yang C, Han Q-m, Ye X, Lei W, Qian W. MNK1 inhibitor CGP57380 overcomes mTOR inhibitor-induced activation of elF4E: the mechanism of synergic killing of human T-ALL cells. Acta Pharmacologica Sinica. 2018; 39: 1894-901.

49. Lineham E, Spencer J, Morley SJ. Dual abrogation of MNK and mTOR: a novel therapeutic approach for the treatment of aggressive cancers. Future Medicinal Chemistry. 2017; 9:1539-55.

50. Yang X, Zhong W, Cao R. Phosphorylation of the mRNA cap-binding protein elF4E and cancer. Cellular signalling. 2020; 73: 109689.

51. Abdelaziz AM, Yu M, Wang S. Mnk inhibitors: a patent review. Pharmaceutical patent analyst. 2021;10:25-35.

52. Zhang $X$, Zhang X, Hu S, Zheng M, Zhang J, Zhao J, et al. Identification of miRNA-7 by genome-wide analysis as a critical sensitizer for TRAIL-induced apoptosis in glioblastoma cells. Nucleic Acids Research. 2017;45:5930-44.

\section{Figures}



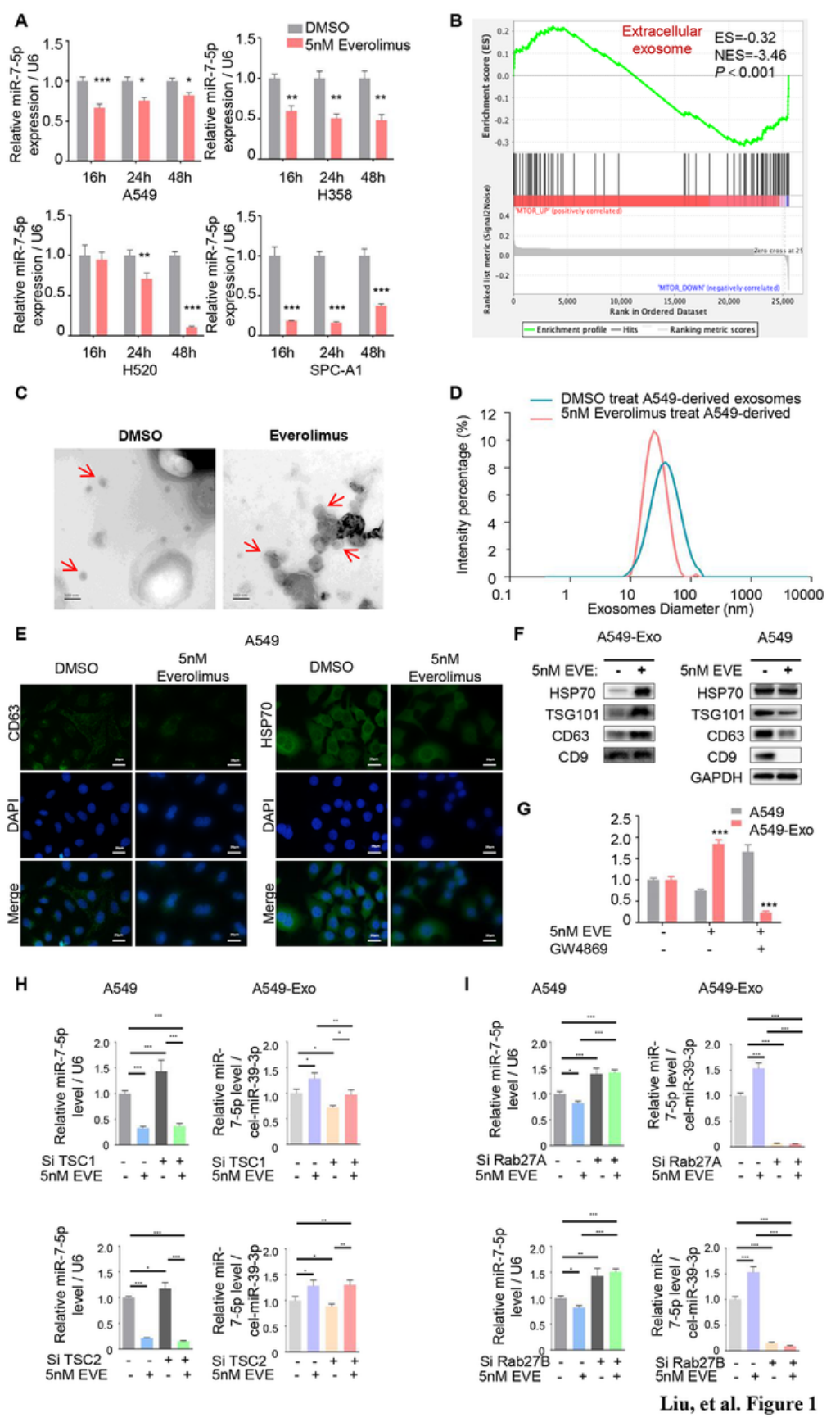

Figure 1

Everolimus targeted mTORC1 inducing NSCLC cells to secrete miR-7-5p-loaded exosomes in Rab27A and Rab27B dependent manners. A. The levels of miR-7-5p in NSCLC cells (A549, H358, H520 and SPC-A1) treated with Everolimus were measured by qPCR for $16 \mathrm{~h}, 24 \mathrm{~h}$ and $48 \mathrm{~h}$. T-test. Each bar represents mean \pm SD. B. GSEA analysis showed the difference gene set between mTROlow and mTORhigh. ES, enrichment score; NES, normalized enrichment score. C. Transmission electron microscopy images for exosomes pointed by the red arrow (scale bar=100 $\mathrm{nm}$ ). D. Size distribution analysis of purified exosomes by Zetasizer Nano ZS90. E. Immunofluorescence was carried out to detect the expression of exosomal marker CD63 (green) in A549 and SPC-A1 cell lines with or without Everolimus treatment. F. Exosomal markers including HSP70, TSG101, CD63 and CD9 were analyzed by Western blotting in A549 cells and A549derived exosomes. DAPI (blue) was used for nuclear staining. G. The levels of intracellular and exosomal miR-7-5p were measured by qPCR. A549 cells were treated with $5 \mathrm{nM}$ Everolimus combined with or without $10 \square \mathrm{M} \mathrm{GW} 4869$ for $24 \mathrm{~h}$. H. A549 cells were transfected with siTSC1/2 and treated with or without Everolimus for 24h. The qPCR detected the levels 
of intracellular and exosomal miR-7-5p in the corresponding cells above. I. A549 cells were transfected with siRab27A/B and treated with or without Everolimus for $24 \mathrm{~h}$. The $\mathrm{qPCR}$ detected the levels of intracellular and exosomal miR-7-5p in the corresponding cells above. ${ }^{*} P<0.05, * \star P<0.01$, ***P $<0.001$.

A
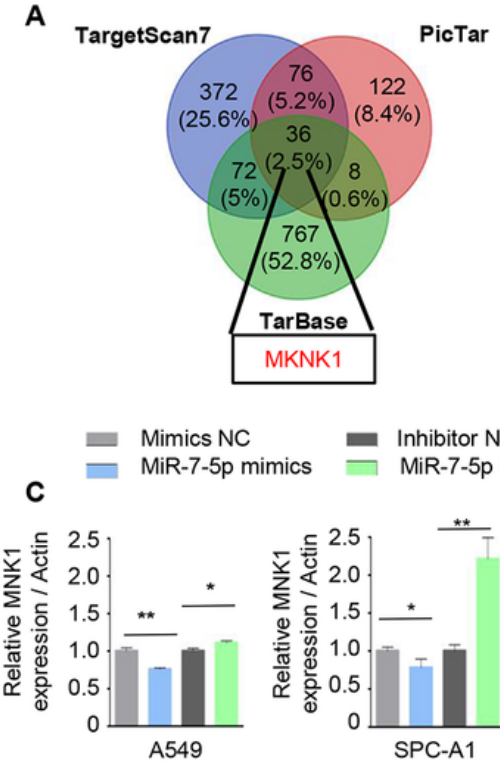

E

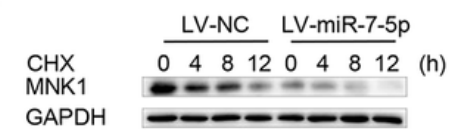

F

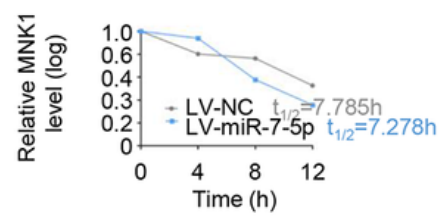

H

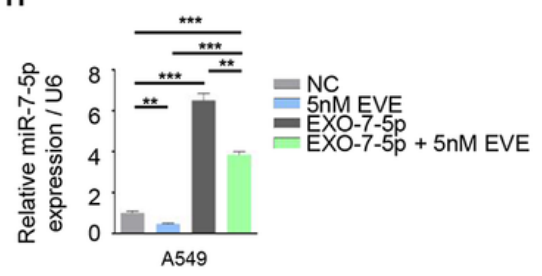

B

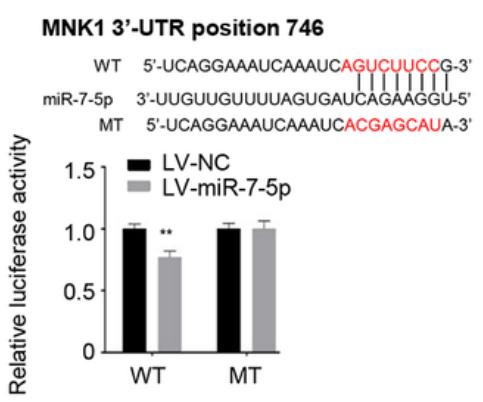

D

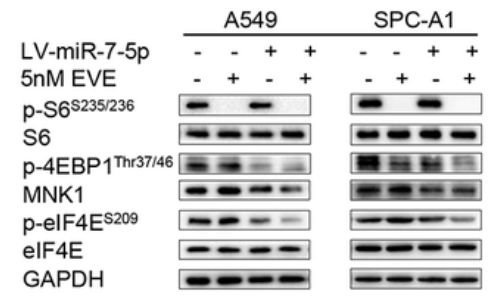

G
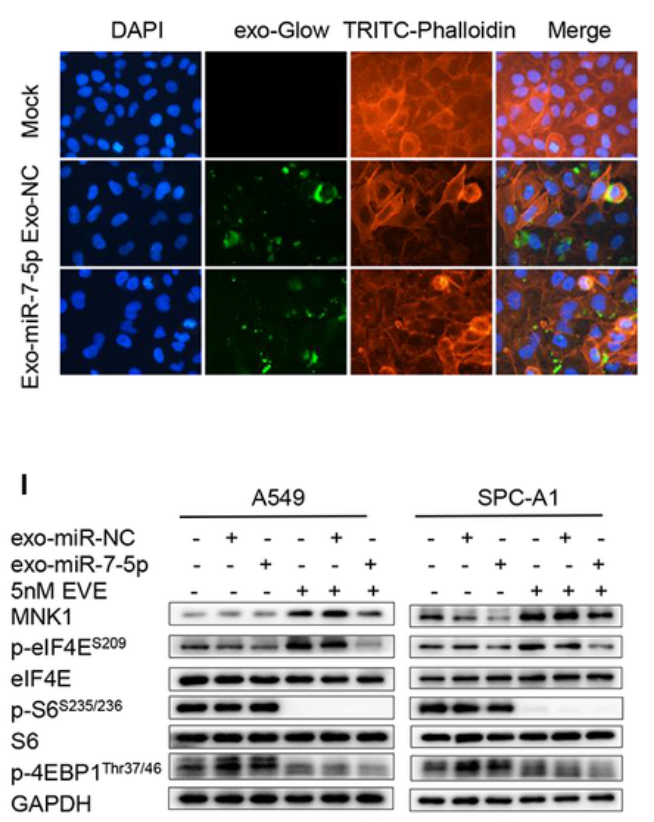

Liu, et al. Figure 2

\section{Figure 2}

Loss of intracellular miR-7-5p induced phosphorylation of MNK/elF4E axis, but supplement of extra exosomal miR-7-5p could reverse it. A. The MNK1 was the target gene of miR-7-5p predicted by the bioinformatics analysis. B. The binding sites of miR-7-5p in 3'-UTR region of MNK1. Wild-type and mutant sequences were indicated (upper). The LV-miR-7-5p and control A549 cells were transfected with a luciferase reporter containing the 3囚-UTR (WT or Mut) of MNK1, indicating MNK1 was the target gene of miR-7-5p. C. Changing the levels of miR-7-5p in A549 and SPC-A1 cells by indicated mimics or inhibitor for 24h, the mRNA of MNK1 were determined by qPCR. D. The LV-miR-7-5p and control A549 and SPC-A1 cells were treated with DMSO or $5 \mathrm{nM}$ Everolimus alone or in combination for $24 \mathrm{~h}$ to detect the expression of MNK1 and p-elF4ES209 and downstream of mTORC1, including p-S6/S6 S235/236 and p-4EBP1Thr37/46, by Western 
blotting. E. The LV-miR-7-5p A549 cells were treated with cycloheximide $(20 \mu \mathrm{g} / \mathrm{mL})$ and collected in the indicated times. The protein of MNK1 was detected by Western blotting. F. The half-life of the MNK1 protein was calculated. G. Fluorescently labeled exosomes derived from indicated A549 cells were internalized by A549 cells (exo-NC means exosomes derived from A549 infected with LV-NC; exo-miR-7-5p means exosomes derived from A549 infected with LVmiR-7-5p). Representative images were filmed after cells were fixed and stained (magnification, $400 \times$ ). H. The miR-7-5p level was detected by qPCR in A549 cells treated with Everolimus or miR-7-5p loaded exosomes alone or in combination. I. The expression of MNK1 and p-elF4ES209 and downstream of mTORC1 including p-S6/S6 S235/236 and p-4EBP1 Thr37/46, in A549 and SPC-A1 cells treated with Everolimus or miR-7-5p loaded exosomes alone or in combination were detected by Western blotting.
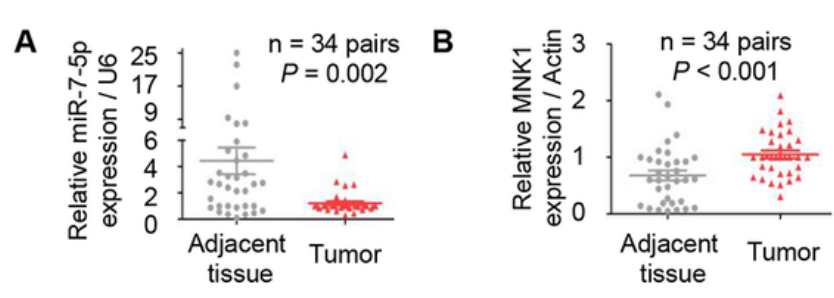

D
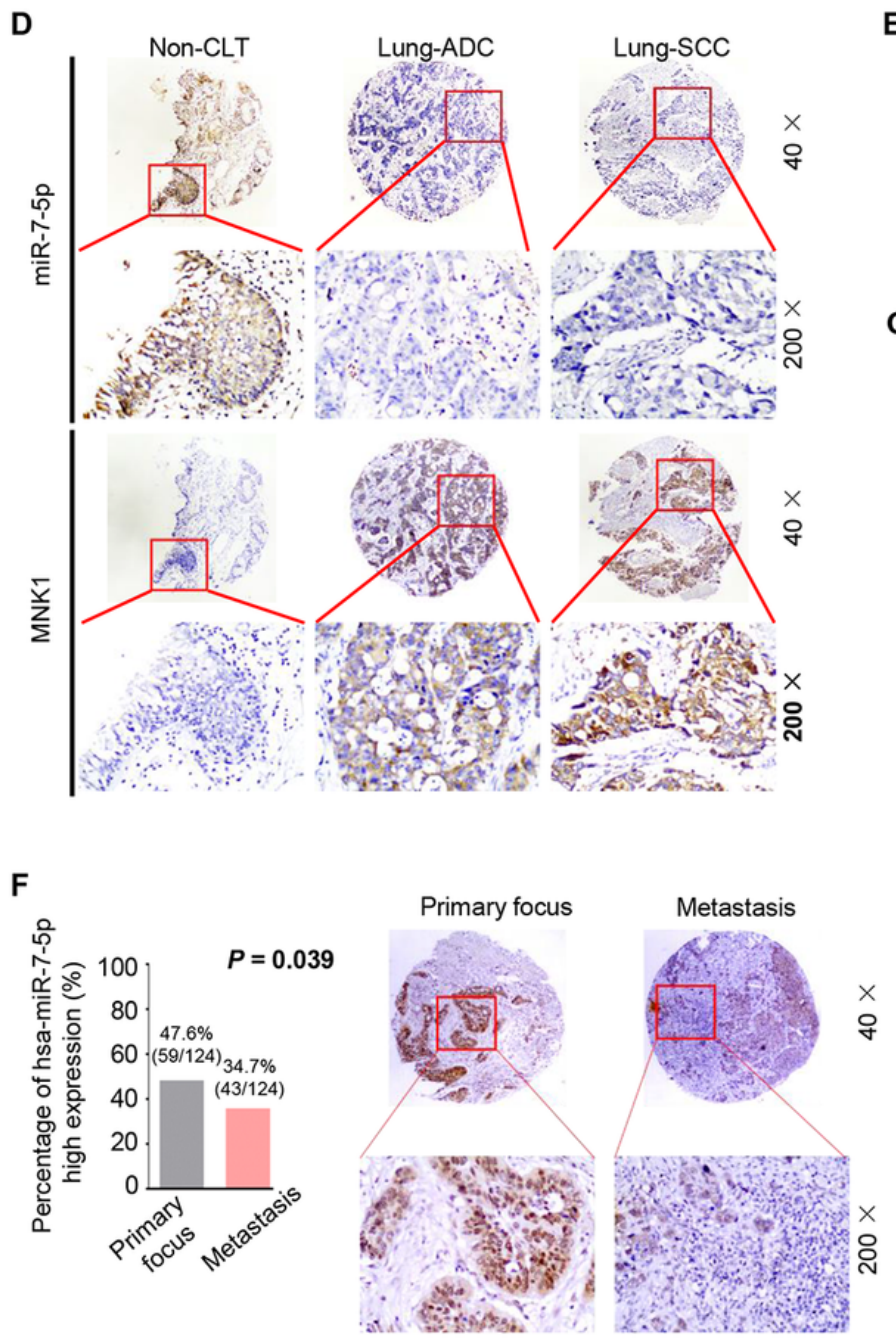

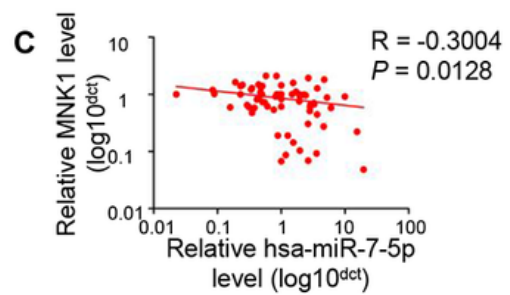

E
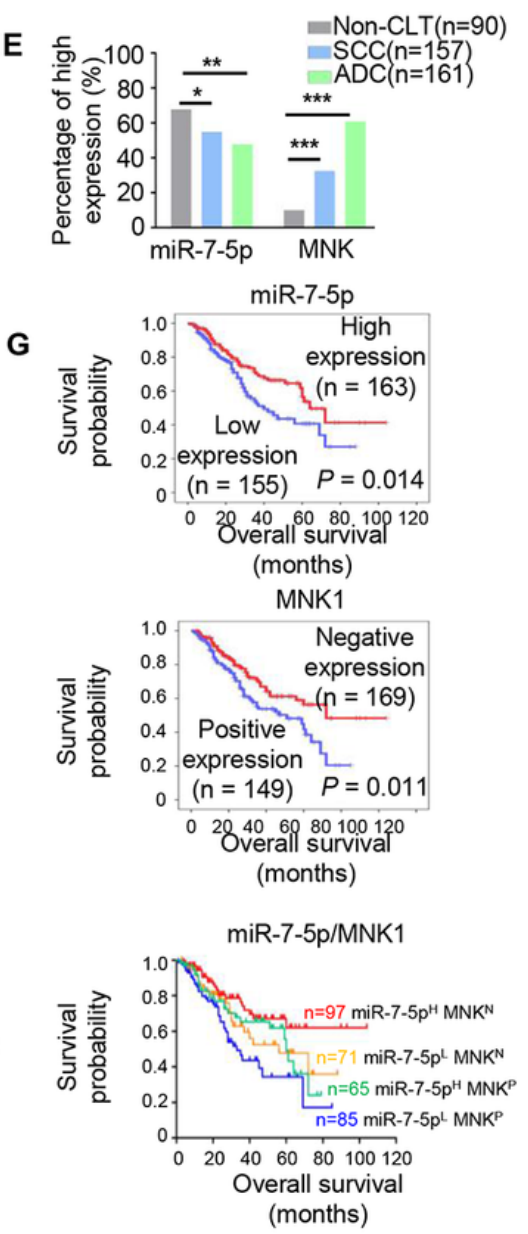

Liu, et al. Figure 3

\section{Figure 3}

The decreased miR-7-5p and elevated MNK1 were associated with poor prognosis of NSCLC. A-B. The level of miR-7-5p and MNK1 mRNA were analyzed by RT-qPCR in 34 pairs of NSCLC tissue samples and corresponding adjacent normal 
lung samples. Statistical significance was calculated using the Wilcoxon test $(P=0.002, P<0.001$, respectively). C. Correlation analysis of miR-7-5p and MNK1 mRNA levels by Spearman's rank correlation coefficient $(R=-0.3004$, $P=0.0128)$. D. In situ hybridization of miR-7-5p and immunohistochemistry staining for MNK1 protein in lung SCC, ADC, and non-cancerous lung tissue microarray, respectively. Both expression of miR-7-5p and MNK1 protein predominantly localized in the cytoplasm or the nucleus. Upper: Original magnification, 40x. Lower: original magnification, 200x. E. The levels of miR-7-5p and the expression of MNK1 in lung SCC and ADC compared to non-CLT. Significant differences were observed between the groups, statistically evaluated by chi-square test. F. Left. The levels of miR-7-5p in metastasis locus of NSCLC compared to primary tumors, statistically evaluated by Chi-square test $(P=0.039)$. Right. Representative ISH staining of miR-7-5p in primary tumors and metastasis locus of NSCLC. Upper: Original magnification, 40×. Lower: original magnification, 200x. G. Kaplan-Meier analysis was used to plot the overall survival curves of 318 NSCLC patients with different expression of miR-7-5p and MNK1, statistical significance was assessed by log-rank test. *P < $0.05, * * \mathrm{P}<0.01$, and $* \star * \mathrm{P}<0.001$ compared with control.
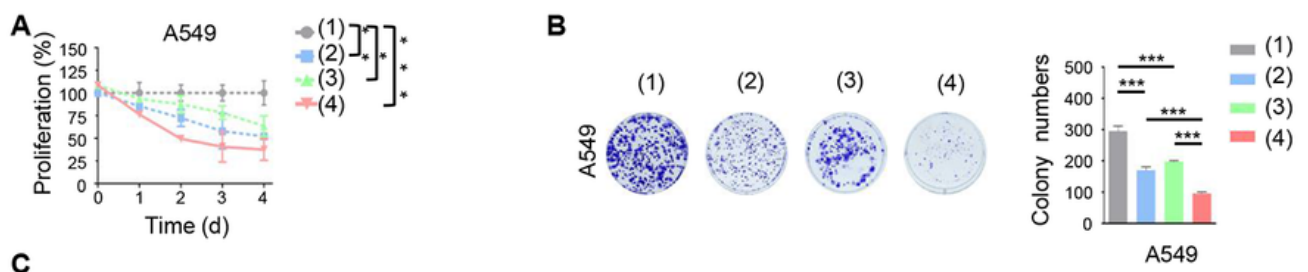

C
(1)
(2)

(3)
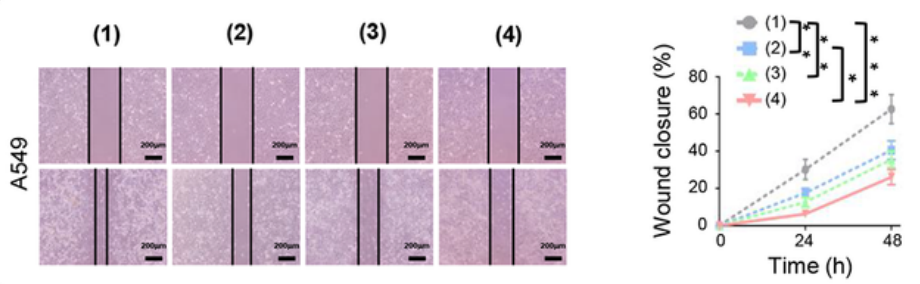

(1) LV-NC

(2) LV-NC + 5nM EVE

(3) LV-miR-7-6p

(4) LV-miR-7-6p + 6nM EVE

(5) PBS

(6) exo-NC

(7) exo-miR-7-5p

D

(1)

(2)

(3)

(4)
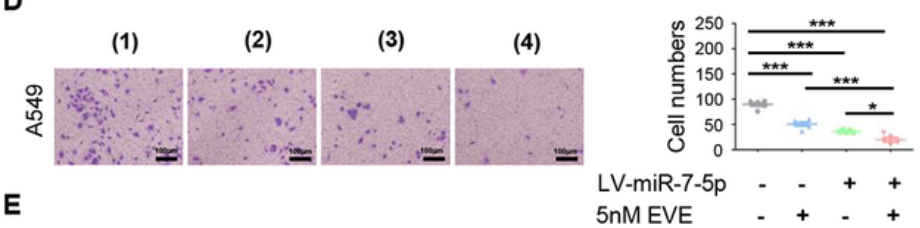

(8) $5 \mathrm{nM}$ EVE

(9) exo-NC + 6nM EVE

(10) exo-miR-7-6p + 5nM EVE

E

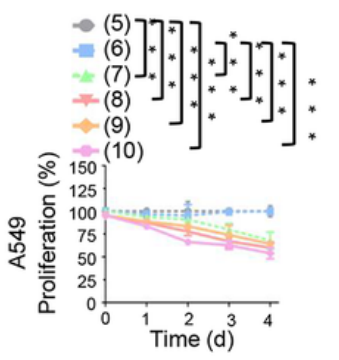
5nMEVE - + - +

G

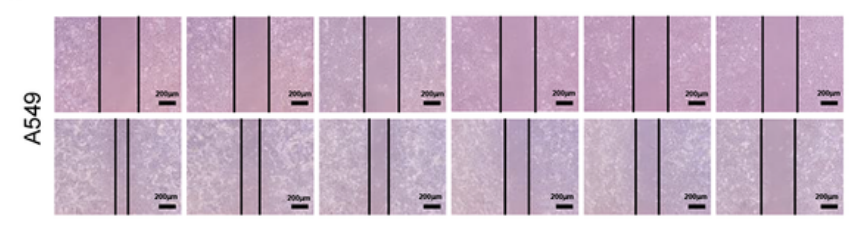

H

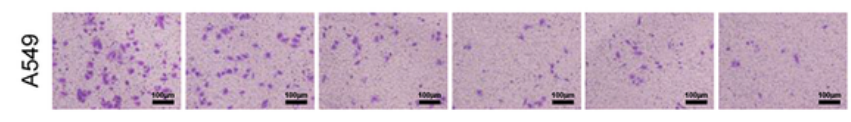

Liu, et al. Figure 4
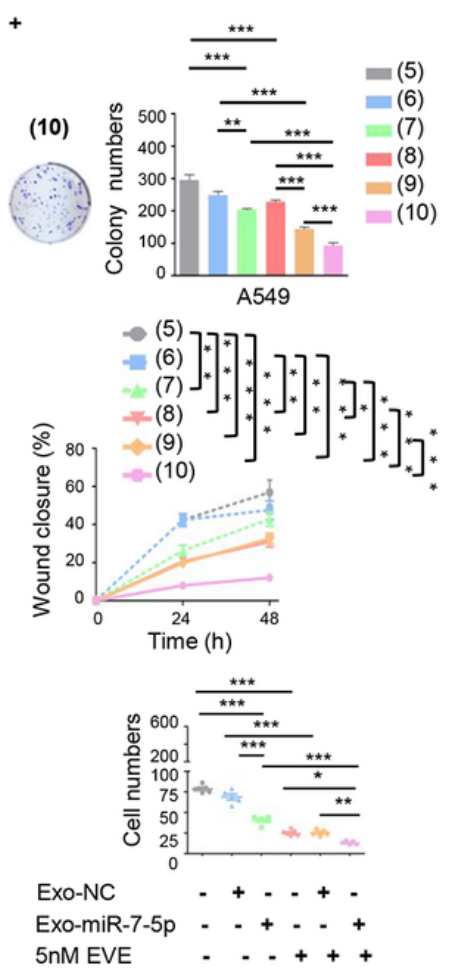
Exosomal miR-7-5p enhanced the anticancer effect of Everolimus in vitro. Indicated A549 cells were treated with or without Everolimus at the settled time. The cell proliferative ability was determined by A. CCK8 assay and B. cell cloneformation assay. The cell migration and invasive ability was determined by $C$. cell scratch test and D. transwell matrigel assay. A549 cells were treated with indicated exosomes or Everolimus for settled times. The cell proliferative ability was determined by E. CCK8 assay and F. cell clone-formation assay. The cell migrating and invasive ability was determined by $G$. cell scratch test and $H$. transwell matrigel assay. Data are shown as mean $\pm S D$. ${ }^{*}<<0.05,{ }^{*} P<0.01$, and ${ }^{* \star *} P<$ 0.001 compared with control.
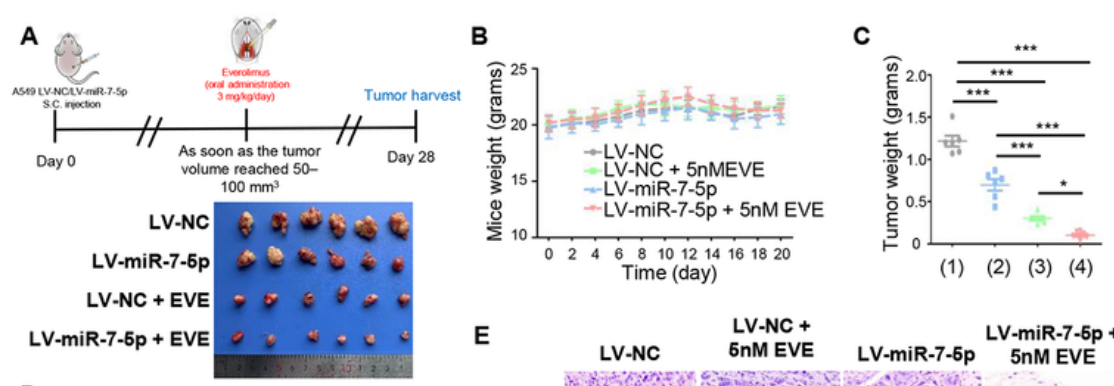

D
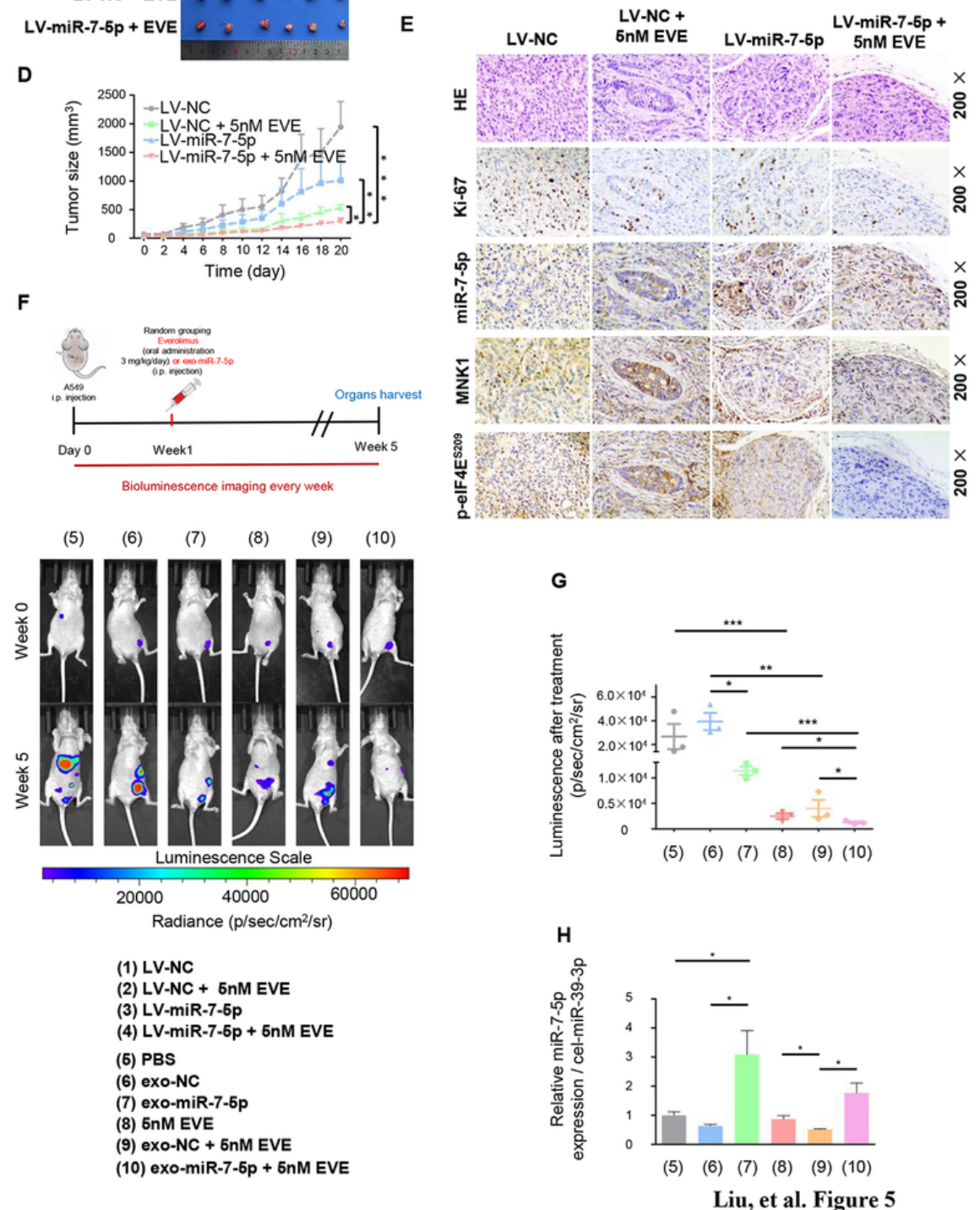

Figure 5

Exosomal miR-7-5p enhanced the anticancer therapeutic efficacy of Everolimus in vivo Five-week-old male nude mice were randomly divided into two groups to establish LV-NC and LV-miR-7-5p A549 cells subcutaneous xenograft nude mice models. As soon as the tumor volume reached $50-100 \mathrm{~mm} 3$, they would be divided into four groups named as 1). the LV-NC group, 2). the LV-miR-7-5p group, 3). the LV-NC combined Everolimus group and 4). the LV-miR-7-5p combined 
Everolimus group. A. Upper: Schematic illustration of Everolimus oral administration for the subcutaneous xenograft nude mice models. Lower: Comparison of tumor engraftment size and weight in nude mice subcutaneously injected into the flanks with indicated A549 cells and indicated treatment. B. The body weight, C. The tumor weight and D. The mice xenograft tumor growth curves of the four groups. E. Immunohistochemical/ In situ hybridization staining for the proliferation marker Ki67, MNK1 and miR-7-5p in the tumor tissues. The A549 cells labeled with luciferase gene were injected into the abdominal cavity of nude mice, and the baseline level of tumor cells was monitored by live imaging technology immediately after injection. One week later, they would be randomly divided into 6 groups for receiving PBS, exo-NC, exo-miR-7-5p, Everolimus, exo-NC combined with Everolimus and exo-miR-7-5p combined with Everolimus treatment, respectively. F. Upper: Schematic illustration of abdominal tumor xenograft nude mice models. Lower: Luminescence signals of intraperitoneal A549-luciferase cells with different treatment groups at the indicated week. Results are shown as the mean \pm SD. G. Analysis of intraperitoneal A549-luciferase cells accepted different treatment groups at the indicated week. $\mathrm{H}$. The levels of exo-miR-7-5p from the plasma of nude mice receiving treatments above were measured by qPCR. * $\mathrm{P}<0.05$, ** $\mathrm{P}<0.01, * \star \star \mathrm{P}<0.001$.
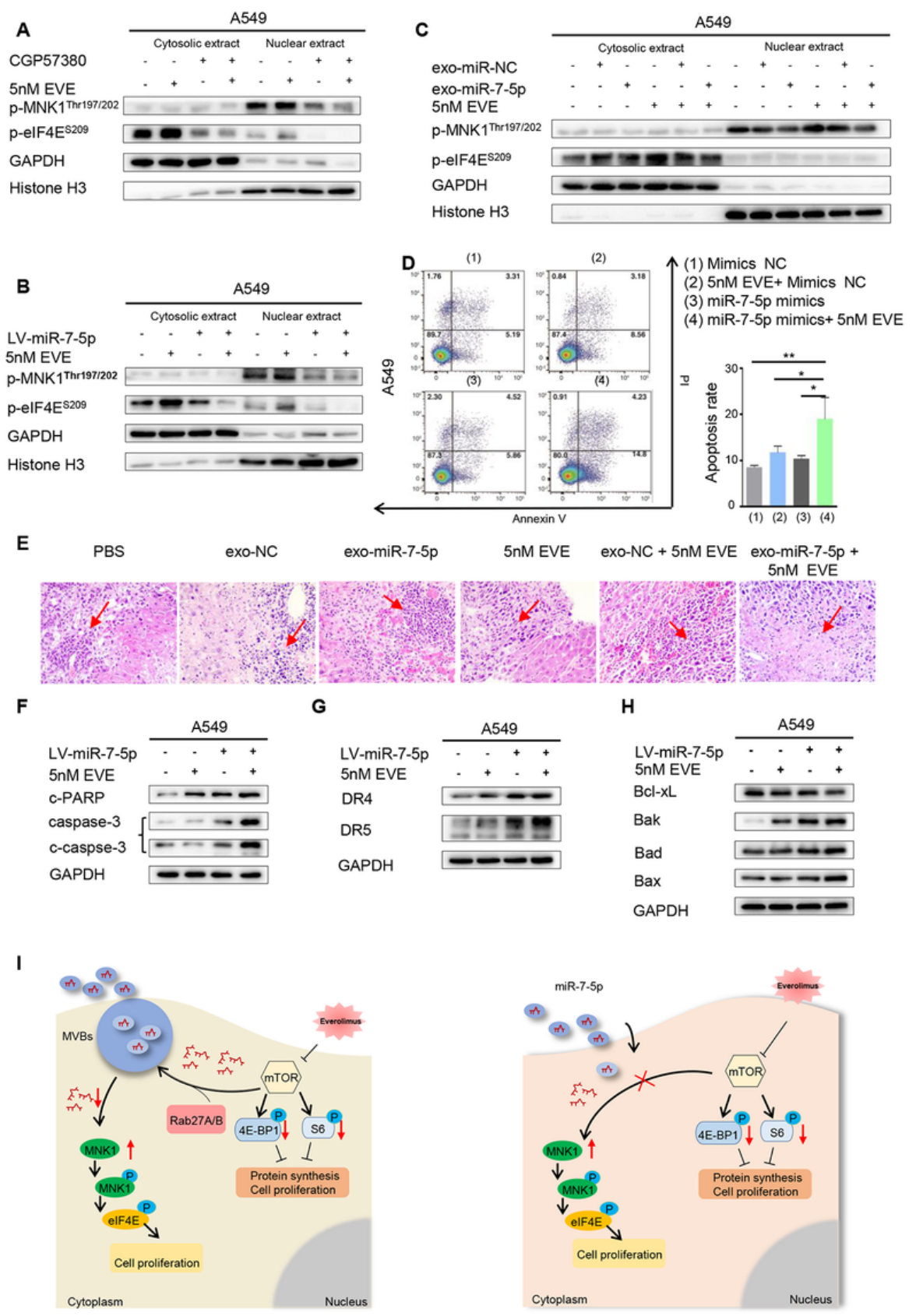

Liu, et al. Figure 6

Page 25/26 


\section{Figure 6}

Combination of miR-7-5p with Everolimus induced apoptosis to exhibit a synergistic anticancer therapeutic efficacy via dual abrogation of MNK/elF4E and mTOR in NSCLC Analysis of proteins of p-MNK1 Thr197/202 and p-elF4ES209 respectively extracted from cytosolic or nuclear in the indicated A549 cells was detected using Western blotting. GAPDH was used as a loading cytoplasmic control. And Histone $\mathrm{H} 3$ was used as a loading nuclear control. A. A549 cells were treated with MNK inhibitor CGP57380 or mTORC1 inhibitor Everolimus alone or in combination. B. The LV-miR-7-5p or LV-NC A549 cells were treated with or without Everolimus. C. Treatment of A549 cells with Everolimus or miR-7-5p loaded exosomes alone or in combination. D. Cells were transfected with indicated mimics with or without Everolimus for $48 \mathrm{~h}$. Apoptotic cells were analyzed by flow cytometry using Annexin V/ PI staining. Columns, means of three replicate determinations; each bar represents mean $\pm S D$. ${ }^{*} P<0.05$, $* \star P<0.01$. E. Representative microscopic images of liver metastatic lesions among all groups in previous abdominal metastasis models. The arrow showed the metastasis. F-H. The stably overexpressed miR-7-5p of A549 cells were treated with Everolimus or DMSO for $48 \mathrm{~h}$. Cell lysates were harvested for western blotting analysis to detect the indicated apoptotic proteins, and GAPDH was used as a loading control. I. Schematic representation of a model for the major molecular mechanisms of "Exosome-mediated miR-7-5p delivery enhances the anticancer effect of Everolimus via blocking MNK/elF4E axis" in NSCLC.

\section{Supplementary Files}

This is a list of supplementary files associated with this preprint. Click to download.

- figureS1.pdf

- Figures2.pdf

- FigureS3.pdf

- FigureS4.pdf

- SupportingInformationJECCR.docx 